\title{
DARK MATTER AND GAS CONCENTRATIONS IN THE NGC 4532/DDO 137 SYSTEM
}

\author{
G. LYLE HOFFMAN \\ Department of Physics, Lafayette College, Easton, PA 18042; hoffmang@nova.phys.lafayette.edu \\ NANYAO Y. LU \\ Infrared Processing and Analysis Center, California Institute of Technology, Mail Stop 100-22, Pasadena, CA 91125; lu@ipac.caltech.edu \\ E. E. SAlpeter \\ Center for Radiophysics and Space Research, Cornell University, Ithaca, NY 14853; salpeter@spacenet.tn.cornell.edu \\ AND \\ BRYAN M. CONNELL \\ Department of Physics, Lafayette College, Easton, PA 18042; connellb@lafayette.edu \\ Received 1998 February 11 ; accepted 1998 October 29
}

\begin{abstract}
H I synthesis mapping of NGC 4532 and DDO 137, a pair of Sm galaxies on the edge of the Virgo Cluster, is used to determine rotation curves for each of the galaxies and to resolve the structure and kinematics of three distinct $\mathrm{H}$ I concentrations embedded in an extended envelope of diffuse $\mathrm{H}$ I discovered in earlier Arecibo studies of the system. The $\mathrm{H}$ I masses of the three concentrations do not appear to be sufficient for them to be self-gravitating; however, their $\mathrm{H}$ I masses and dynamical masses are very similar to those of faint Im galaxies in the Virgo Cluster. The peak H I column density of each of the clouds is close to the star formation threshold, but CCD images in $B$ and $R$ reveal no trace of stars or star formation. If the system is gravitationally bound and in virial equilibrium, we find its total mass to be 10 times that within the outermost $\mathrm{H}$ I contours of the individual galaxies and clouds. Thirtyseven percent of the total $\mathrm{H}$ I lies outside the boundaries of the individual galaxies as determined by the VLA mapping; $28 \%$ cannot be ascribed to either galaxy or to the discrete concentrations. One possible way to account for the velocity field and the large quantity of diffuse gas is to assume that DDO 137, the gas concentrations, and other apparent tidal features are due to gas infall into clumps within the dark matter potential of the group, and that prior to the interaction the group consisted of an irregular galaxy (NGC 4532) and a large, star-poor H I cloud like H i 1225+ 01.

Key words: galaxies: individual (NGC 4532, DDO 137) - galaxies: irregular - galaxies: photometry intergalactic medium - quasars: absorption lines - radio emission lines
\end{abstract}

\section{INTRODUCTION}

Studies of galaxy pairs and small groups in many cases reveal extended bridges, streams, and detached clouds of $\mathrm{H}$ I at considerable distances from the stellar components of the interacting galaxies (e.g., Haynes, Giovanelli, \& Roberts 1979; Haynes 1981; van Moorsel 1988; van Driel et al. 1992; Chengalur, Salpeter, \& Terzian 1994; Li \& Seaquist 1994; Yun, Ho, \& Lo 1994; Elmegreen et al. 1995a; Hibbard \& van Gorkom 1996; Kaufman et al. 1997; Nordgren et al. 1997a, 1997b; earlier references are cited in each of those papers). The streams and clouds are generally assumed to be generated tidally from galaxies that were previously well separated, and dynamical modelling (Toomre \& Toomre 1972; Howard et al. 1993; Sofue 1994; Elmegreen et al. 1995b; Dubinski, Mihos, \& Hernquist 1996; Mihos \& Hernquist 1996; Barnes \& Hernquist 1996; Mihos, Dubinski, \& Hernquist 1998; earlier work is reviewed by Barnes \& Hernquist 1992a) generally confirms that the tidal assumption is plausible, although the simulations do not usually achieve the pronounced distension of the $\mathrm{H}$ I relative to the stars that is often seen in the observed cases (see, however, Smith, Struck, \& Pogge 1997).

It has been suggested that some Ly $\alpha$ absorption lines, especially the Lyman limit systems (LLSs), arise from tidally distended gas around individual galaxies (van Gorkom 1993) or within groups of galaxies (Morris \& van den Bergh 1994; Mo \& Morris 1994). Such absorption systems account for a few percent of the low-redshift Ly $\alpha$ absorptions observed with the Hubble Space Telescope (HST) (Storrie-Lombardi et al. 1994; Stengler-Larrea et al. 1995; Bahcall et al. 1996); whereas low-redshift Ly $\alpha$ forest systems are found in galaxy groups with a covering factor near unity (Le Brun, Bergeron, \& Boissé 1996; Bowen, Blades, \& Pettini 1996; Morris et al. 1993), LLSs are found much more rarely. The $\mathrm{H} \mathrm{I}$ in interacting systems in any case serves as a historical record allowing the trajectories of the interacting galaxies to be traced back in time in some cases (Phookun et al. 1992; Chengalur, Salpeter, \& Terzian 1995; Elmegreen et al. 1995b). Condensations of gas within the $\mathrm{H}$ I tails appears in some instances to form new dwarf galaxies (Mirabel, Dottori, \& Lutz 1992; Elmegreen, Kaufman, \& Thomasson 1993; Duc \& Mirabel 1994; Hibbard et al. 1994; Hunsberger, Charlton, \& Zaritsky 1996; Malphrus et al. 1997).

On the other hand, not all diffuse, extended $\mathrm{H}$ I clouds appear to have been generated tidally from previously more compact galaxies. Tidal effects may have shaped the Leo ring, but the progenitor does not appear to have been any of the optically identified galaxies in the group (Schneider 1989). The large, diffuse cloud H I 1225+01 (Giovanelli, Williams, \& Haynes 1991) appears to be well isolated from any galaxy large enough to have exerted tidal influence. In both cases, the $\mathrm{H}$ I gas appears to have never been part of any optically bright galaxy, and its dynamics appears to be 
TABLE 1

Optical Details

\begin{tabular}{ccccccccr}
\hline \hline Galaxy & R.A. (B1950.0) & Decl. (B1950.0) & Type & $\begin{array}{c}V_{\odot} \\
\left(\mathrm{km} \mathrm{s}^{-1}\right)\end{array}$ & $\begin{array}{c}\text { Distance } \\
(\mathrm{Mpc})\end{array}$ & $\begin{array}{c}B_{T}^{0} \\
(\mathrm{mag})\end{array}$ & $\begin{array}{c}M_{B} \\
(\mathrm{mag})\end{array}$ & $\begin{array}{c}D_{25} \\
(\mathrm{kpc})\end{array}$ \\
\hline NGC $4532 \ldots \ldots$ & 123146.8 & 064443 & Sm III & 2154 & 19 & 11.94 & -19.45 & 15.6 \\
DDO $137 \ldots \ldots$ & 123212.1 & 063441 & Sm IV & $\ldots$ & 19 & 14.58 & -16.81 & 7.1 \\
\hline
\end{tabular}

NotE.- Units of right ascension are hours, minutes, and seconds, and units of declination are degrees, arcminutes, and arcseconds.

controlled by dark matter potential wells of galaxy group scale rather than by tides due to individual galaxy halos (see also Hoffman et al. 1998).

NGC 4532 and DDO 137 were initially mapped by us at Arecibo $^{1}$ as two distinct irregular galaxies (Helou, Hoffman, \& Salpeter 1984; Hoffman et al. 1987), but a chance reference beam for an unrelated observation led to our discovery of a large, diffuse cloud of $\mathrm{H}$ I surrounding the pair (Hoffman et al. 1992, 1993). Presumably, if $H S T$ spectra were obtained for any quasi-stellar objects (QSOs) lying behind the extended $\mathrm{H}$ I cloud, anywhere within the outermost contours in our Arecibo map (see Fig. 2 below), they would exhibit LLSs. Indications from the Arecibo maps of condensations within the diffuse cloud, but well outside the optical images of the two galaxies, motivated the Very Large Array (VLA) ${ }^{2}$ maps presented here. A desire for finer spatial resolution of the two main galaxies was another motivating factor. CCD imaging was undertaken to see if any low surface brightness (LSB) optical features could be found in the condensations within the diffuse cloud, and to investigate the star formation histories of the two galaxies. As we shall detail below, we do not think that tides acting on the $\mathrm{H}$ I reservoirs of two initially well-separated irregular galaxies can account for the diffuse $\mathrm{H}$ I envelope. This system seems to be another example of a primordial, starpoor cloud of $\mathrm{H}$ I reacting to a clumpy group scale dark matter halo.

Optical details culled mainly from de Vaucouleurs et al. (1991, hereafter RC3) are presented in Table 1, which lists for each member galaxy the coordinates (epoch B1950.0) of the nucleus; the morphological type as coded by Binggeli (1985); the optical heliocentric velocity; our assumed distance, taken to be the same as the Virgo Cluster core ; the blue apparent magnitude $B_{T}^{0}$ corrected for inclination and Milky Way extinction (from RC3); the blue absolute magnitude determined from that assumed distance and $B_{T}^{0}$; and the optical diameter at the $25 \mathrm{mag} \mathrm{arcsec}^{-2}$ isophote.

A brief description of the CCD imaging is given in $\S 2$. Details and results of our $\mathrm{H}$ I synthesis mapping are given in $\S 3$. In $\S 4$, we discuss the dynamics and possible evolutionary scenarios for the system. We end with a summary and conclusions in $\S 5$.

\section{CCD IMAGING}

Both NGC 4532 and DDO 137 were observed in $U, B, V$, $R$, and $I$ with the Palomar 60 inch $(1.5 \mathrm{~m})$ telescope equipped with a TK $2048 \times 2048$ CCD chip under photo-

\footnotetext{
${ }^{1}$ The Arecibo Observatory is part of the National Astronomy and Ionosphere Center, which is operated by Cornell University under a management agreement with the National Science Foundation.

2 The Very Large Array is operated by the National Radio Astronomy Observatory, which is a facility of the National Science Foundation, operated under cooperative agreement by Associated Universities, Inc.
}

metric conditions. For NGC 4532, we also obtained an $\mathrm{H} \alpha$ image. These images will be fully described in a later paper in which we study the past and current star formation activities in these galaxies (Lu, Hoffman, \& Salpeter 1998, hereafter LHS98). Here, we make use of the $B$ and $R$ images only. With a pixel size of 0.742 , the CCD field is large enough for us to put two of the three $\mathrm{H}$ I concentrations of the system (i.e., clouds A and B; see $\S 3.3$ ) on the CCD image of DDO 137, with cloud C on the CCD frame for NGC 4532. The deep $R$ image of DDO 137 has an rms sensitivity of $\sim 26 \mathrm{mag} \operatorname{arcsec}^{-2}$. But at this level, none of the $\mathrm{H} \mathrm{I}$ clouds is detected optically.

Elliptical contours are fitted to each of the galaxy images to extract a surface brightness profile, following the prescription given by Lu et al. (1993). However, the inner parts of both galaxies are dominated by the light from a few clumps that are located off the galaxy center as determined by the isophotes at large radii. As a result, the fitted contours are dictated by these clumps at small radii, causing the centers of the ellipses to shift. To avoid this problem, we adopted a hybrid method. Namely, the fitted result was used only at radii larger than $r_{c}$ where the surface brightness is smooth; but at radii less than $r_{c}$ the radial surface brightness profile was determined by using a series of concentric elliptical contours with fixed center position, shape, and position angle equal to the corresponding mean values of the fit between radii $r_{1}$ to $r_{2}$ in the outer galaxy. Note that the two latter parameters were also averaged over the $B$ and $R$ passbands. In this "concentric elliptical model" method, the surface brightness along an ellipse of radius $r_{n}$ is given by the mean value within the annulus between radii $0.5\left[r_{n}+r_{(n-1)}\right]$ and $0.5\left[r_{n}+r_{(n+1)}\right]$, where $r_{(n-1)}, r_{n}$ and $r_{(n+1)}$ are the radii of three successive ellipses. The resulting hybrid surface brightness profiles are shown in Figure 8 below for NGC 4532 and DDO 137.

For each galaxy, Table 2 gives the values of $r_{c}, r_{1}$, and $r_{2}$, as well as a few parameters derived from the fit to the galaxy

TABLE 2

CCD PHOTOMETRY

\begin{tabular}{ccc}
\hline \hline Parameter & N4532 & DDO 137 \\
\hline$r_{c}(\operatorname{arcsec}) \ldots \ldots \ldots \ldots \ldots \ldots$. & 50 & 30 \\
$r_{1}(\operatorname{arcsec}) \ldots \ldots \ldots \ldots \ldots \ldots \ldots$ & 60 & 45 \\
$r_{2}(\operatorname{arcsec}) \ldots \ldots \ldots \ldots \ldots \ldots$ & 110 & 75 \\
$B_{T}(\mathrm{mag}) \ldots \ldots \ldots \ldots \ldots \ldots \ldots$ & $12.15 \pm 0.02$ & $14.76 \pm 0.04$ \\
$(B-R)_{T}(\mathrm{mag}) \ldots \ldots \ldots \ldots \ldots$ & $0.71 \pm 0.05$ & $0.95 \pm 0.07$ \\
$D_{25}(\mathrm{~B})(\operatorname{arcmin}) \ldots \ldots \ldots \ldots$ & $3.12 \pm 0.10$ & $1.23 \pm 0.07$ \\
$D_{26}(\mathrm{~B})(\operatorname{arcmin}) \ldots \ldots \ldots \ldots$. & $3.70 \pm 0.11$ & $1.86 \pm 0.11$ \\
Ellipticity $\ldots \ldots \ldots \ldots \ldots \ldots \ldots$ & $0.585 \pm 0.03$ & $0.250 \pm 0.03$ \\
P.A. $(\operatorname{deg}) \ldots \ldots \ldots \ldots \ldots \ldots$ & $159 \pm 1$ & $130 \pm 3$ \\
$\mu_{0}(\mathrm{~B})\left(\mathrm{mag} \operatorname{arcsec}{ }^{-2}\right) \ldots \ldots$. & $19.77 \pm 0.1$ & $23.71 \pm 0.2$ \\
$r_{s}(\mathrm{~B})(\operatorname{arcsec}) \ldots \ldots \ldots \ldots \ldots \ldots$ & $19.39 \pm 0.5$ & $26.49 \pm 1.0$ \\
\hline
\end{tabular}


disk between $r_{1}$ and $r_{2}$ : the total magnitude in $B, B-R$ color, the $B$-band isophotal diameters at $B=25$ and 26 mag $\operatorname{arcsec}^{-2}$, the mean ellipticity and position angle averaged over the $B$ and $R$ passbands, the $B$-band exponential scale length $r_{s}$ as defined in $\mu(r)=\mu_{0}+1.086 r / r_{s}$, and the $B$-band central surface brightness $\mu_{0}$. If appropriate, a roughly estimated rms uncertainty is also listed.

\section{NEUTRAL HYDROGEN OBSERVATIONS}

The $\mathrm{H}$ I mapping for these galaxies was conducted at the Very Large Array in two observing sessions as detailed in Table 3, which lists for each source the date of the observing run, the pointing center (epoch B1950.0), the heliocentric velocity to which the receivers were tuned, the array configuration, the number of spectral channels and channel separation in velocity units, and the time spent on source during the observations, not including time spent on calibrators. We have also listed the resulting beam size in arcseconds, the rms noise per channel, and the $3 \sigma$ detection limit for H I column density in each channel. We obtained both $\mathrm{R}$ and $\mathrm{L}$ circularly polarized data. We found, however, that we could not eliminate striping of the C or D array maps as long as the $\mathrm{R}$ polarization data was retained, so we made maps using L polarization only. On-line Hanning smoothing was employed throughout, and the observations were calibrated using sources from the VLA calibrator list. Calibration and data editing were accomplished using standard AIPS tasks. Continuum was subtracted in the $u-v$ domain using the task UVBAS, and maps were made and CLEANed using IMAGR with zero-spacing fluxes as discussed below and with robustness parameter 0 for the best compromise between spatial resolution and maximum signal-to-noise ratio. After imaging, each data cube was corrected for the VLA primary beam.

We estimated zero-spacing fluxes from the Arecibo mapping by interpolating each spectrum between Arecibo velocity channels to find intensities at the velocities of the VLA channels. Then we integrated over the entire spatial map at each interpolated velocity to produce the zerospacing flux estimate for each VLA channel. The issue of what weight is appropriate for zero-spacing fluxes in IMAGR does not appear to have been resolved yet, so we experimented with a wide range of weights. We found that if the weight is made too large, the synthesized beam increases in size. On the other hand, large weights would give us the best chance of recovering all the flux detected in the Arecibo map. The weight we chose is the largest weight that does not increase the beam size by more than $1 \%$, and the resulting spectrum integrated over the VLA map agrees well with the global spectrum integrated over the Arecibo results.

Following common practise, we have abbreviated the names so that the field "N4532" is centered on the optical image of NGC 4532, while "D137" is centered a few arcminutes west of the optical image of DDO 137 in order to give maximum sensitivity to the $\mathrm{H}$ I plume extending to the west of the galaxy as seen in our Arecibo mapping (Hoffman et al. 1992, 1993). The field "N4532/D137" is centered in the region between the two galaxies to provide maximum sensitivity to $\mathrm{H}$ I condensations within that region of the cloud enveloping the two galaxies.

We obtained data for two fields using the D array, one field centered on NGC 4532 and the second nearly due south, a few arcminutes west of the optical image of DDO 137. Since significant emission seems to be confined to the region of overlap in the two D array fields, we combined them as follows: Each $u-v$ data set was first shifted to a common tangent point using AIPS task UVFIX; the sets were then combined using task DBCON. IMAGR was run on the combined data set, producing maps with a $46^{\prime \prime} \times 42^{\prime \prime}$ synthesized beam, $1.6 \mathrm{mJy}$ beam $^{-1} \mathrm{rms}$, and a $3 \sigma$ column density limit of $2.8 \times 10^{19}$ atoms cm $\mathrm{cm}^{-2}$. Finally, we applied a primary-beam correction by averaging the standard VLA primary-beam corrections for each original pointing center, giving each equal weight. The result is a data cube that exhibits the same column densities near the centers of each galaxy but which shows the diffuse emission more clearly than either of the separate fields. We similarly combined the $\mathrm{D}$ array and $\mathrm{C}$ array data sets, weighting the primary-beam corrections by the number of visibilities in each data set. The result is a final set of maps with an 18 ".5 $\times 16^{\prime \prime} 7$ synthesized beam and rms noise $0.58 \mathrm{mJy}^{\text {beam }}{ }^{-1}$ per channel, or a $3 \sigma$ column density limit of $6.5 \times 10^{19}$ atoms cm $\mathrm{cm}^{-2}$. The $\mathrm{C}+\mathrm{D}$ array mosaic is shown in Figure 1, where we have integrated over consecutive sets of three channels. Earlier attempts to merge the data sets using the maximum entropy method task VTESS produced a large smear of apparently spurious emission across the center of the field; that is why we resorted to the method described above. Efforts at merging both VLA data sets with our single-dish Arecibo map (Hoffman et al. 1993, hereafter HLS + 93) are underway (Connell 1998), but our thrust here is to consider the VLA and Arecibo data in parallel.

In addition to emission from each of the two optical galaxies, we have clearly detected emission from three gas concentrations distinct from those galaxies (but still embed-

TABLE 3

Very Large Array ObServations

\begin{tabular}{|c|c|c|c|}
\hline Parameter & N4532 & DDO 137 & N4532/D137 \\
\hline Date.... & 1992 Aug 29 & 1992 Aug 29 & 1994 Dec 8 \\
\hline ................... & 123146.8 & 123152.0 & 123159.6 \\
\hline Decl. & 064443 & 063200 & 063942 \\
\hline$V_{\odot}\left(\mathrm{km} \mathrm{s}^{-1}\right) \ldots \ldots \ldots \ldots \ldots \ldots \ldots \ldots$ & 2000 & 2000 & 2000 \\
\hline Array $\ldots \ldots \ldots \ldots \ldots \ldots \ldots \ldots \ldots \ldots$ & $\mathrm{D}$ & $\mathrm{D}$ & $\mathrm{C}$ \\
\hline Number of channels ............... & 63 & 63 & 63 \\
\hline Channel separation $\left(\mathrm{km} \mathrm{s}^{-1}\right) \ldots \ldots$ & 10.4 & 10.4 & 10.4 \\
\hline Time on source (minutes) .......... & 281 & 278 & 322 \\
\hline Beam $(\operatorname{arcsec}) \ldots \ldots \ldots \ldots \ldots \ldots \ldots$ & $51 \times 45$ & $49 \times 46$ & $15 \times 14$ \\
\hline rms $\left(\mathrm{mJy}\right.$ beam $\left.^{-1}\right) \ldots \ldots \ldots \ldots \ldots \ldots$ & 2.3 & 2.1 & 0.73 \\
\hline $3 \sigma$ limit $\left(10^{19}\right.$ atoms $\left.\mathrm{cm}^{-2}\right) \ldots \ldots$. & 3.5 & 3.2 & 11.4 \\
\hline
\end{tabular}




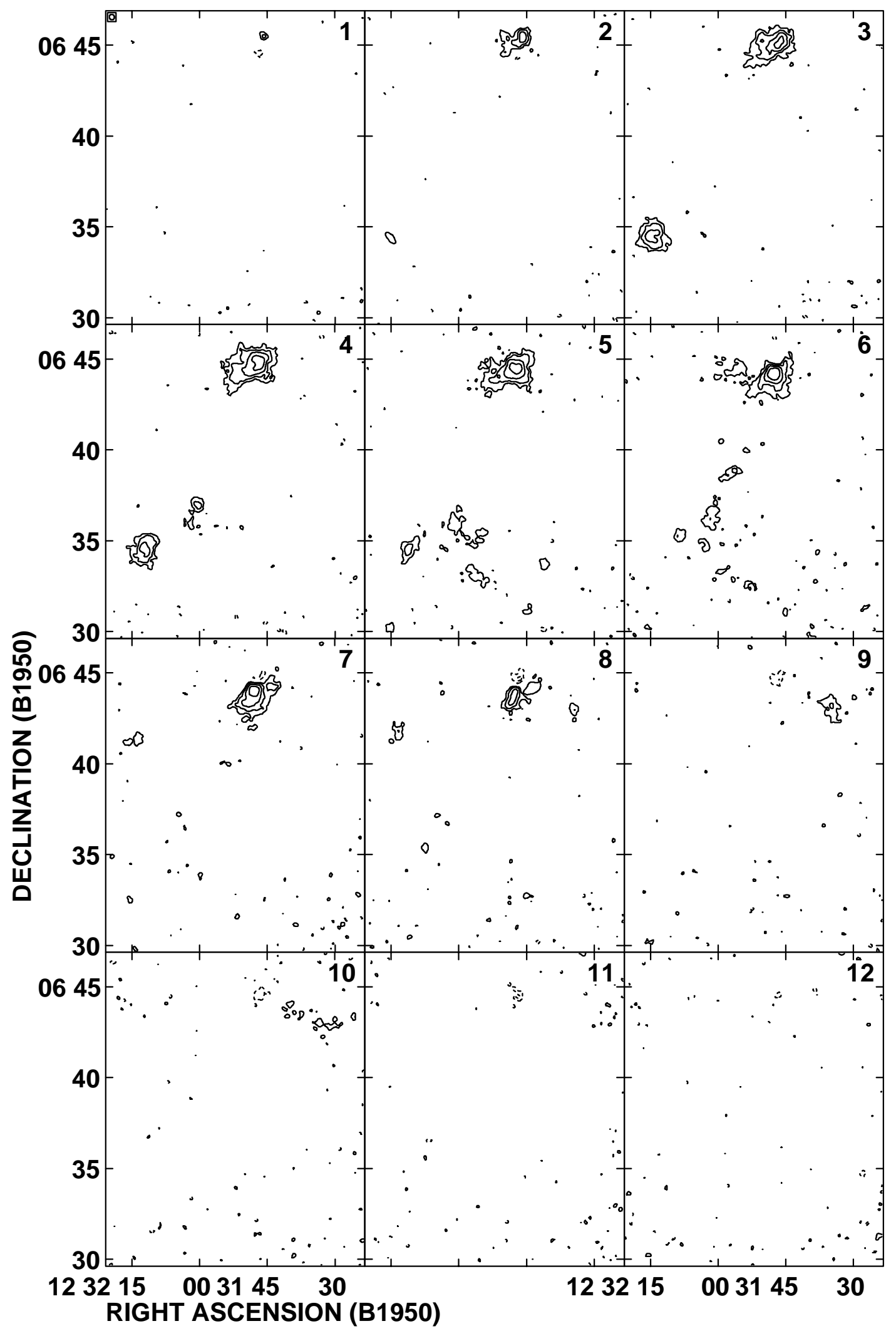

FIG. 1.-Mosaic of three-channel integrated contour maps for the merged C and D array data sets of the galaxy pair NGC 4532/DDO 137. The beam is shown in the top left corner of the top left panel. Each panel consists of the integral over three channels of the data cube; the velocities of the middle channel in each panel, in the order in which they are numbered, are 2146, 2115, 2084, 2052, 2021, 1990, 1958, 1927, 1896, 1864, 1833, and 1802 km s ${ }^{-1}$. Contours are drawn at $-3.6,-1.8,1.8,3.6,7.2$, and $14.3 \times 10^{20}$ atoms cm $\mathrm{cm}^{-2}$. 


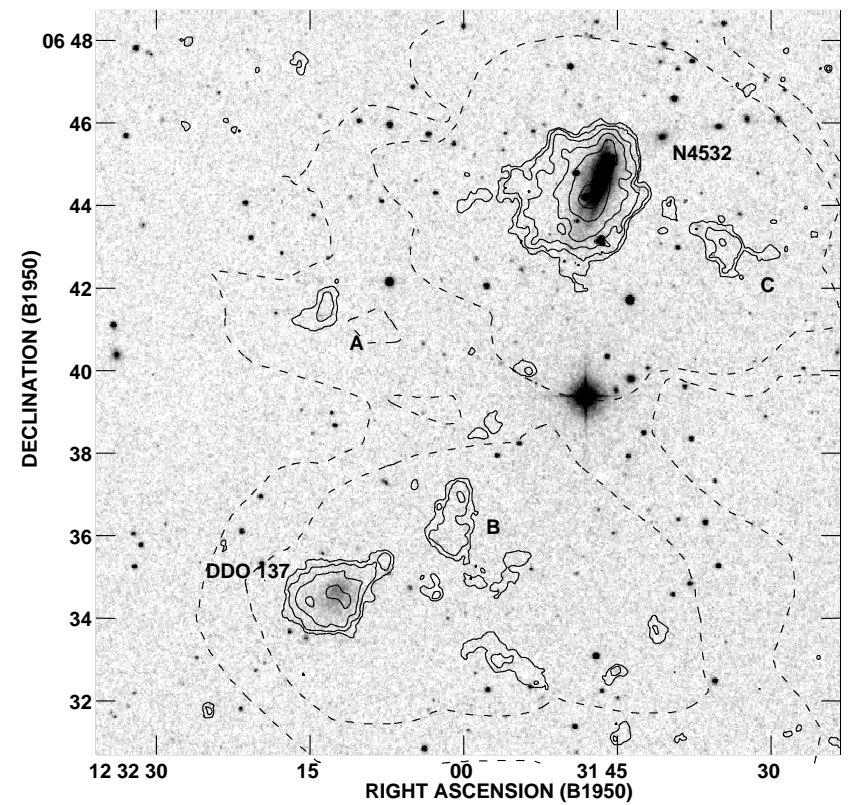

Fig. 2.- Contours of total hydrogen emission from the area around NGC 4532 and DDO 137, integrated over all line-bearing channels for the $\mathrm{C}+\mathrm{D}$ array combination and superposed on a gray-scale image from DSS. Contour levels from the VLA mapping (solid line) are 1.8, 3.6, 7.2, $14.3,28.6$, and $57.2 \times 10^{20}$ atoms $\mathrm{cm}^{-2}$. The dashed curves show the outermost contour levels from the Arecibo map, at $2 \times 10^{19}$ and $4 \times 10^{19}$ atoms $\mathrm{cm}^{-2}$. Three prominent concentrations of gas outside the main galaxies are indicated.

ded within the low column density envelope we found in our Arecibo mapping [HLS + 93]). We will call these clouds A, B, and C as labeled in Figure 2, where the 0th moment map for the entire region is superposed on a Digitized Sky Survey (DSS) ${ }^{3}$ image. Cloud B is part of the extended plume stretching west and south from DDO 137 in the Arecibo map (HLS + 93), which is evident as low-

${ }^{3}$ The Digitized Sky Surveys were produced at the Space Telescope Science Institute under US government grant NAGW-2166. The images of these surveys are based on photographic data obtained using the Oschin Schmidt Telescope on Mount Palomar, which is operated by the California Institute of Technology and the Palomar Observatory. The plates were processed into the present compressed digital form with the permission of that institution.

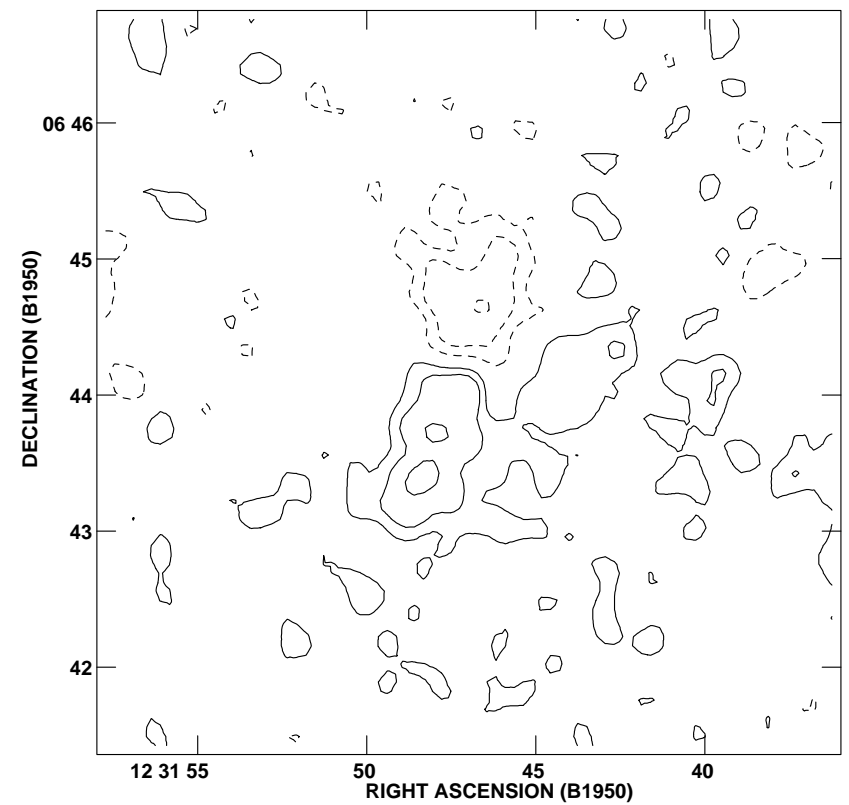

FIG. 3.-Absorption feature at the center of NGC 4532 integrated over velocity for the channels in which it appears in the combined C $+\mathrm{D}$ array data cube. The emission in those channels (excluding all channels where no absorption is seen) is shown with solid contours, absorption with dashed contours. The contour levels are $-0.40,-0.20,-0.10,0.10,0.20$, and 0.40 Jy beam ${ }^{-1} \mathrm{~km} \mathrm{~s}^{-1}$.

level emission in each cube at velocities $1990-2052 \mathrm{~km} \mathrm{~s}^{-1}$. Weak absorption against a central source in NGC 4532 is evident at velocities $\leq 1979 \mathrm{~km} \mathrm{~s}^{-1}$; the feature is shown integrated over velocity in Figure 3.

\subsection{NGC 4532}

The total H I map, velocity field, and second-moment map of the Sm III galaxy NGC 4532 are shown in Figures $4 a, 5 a$, and $6 a$. The total $\mathrm{H} \mathrm{I}$ emission, integrated over the merged C + D array map, we find $50.9 \mathrm{Jy} \mathrm{km} \mathrm{s}^{-1}$, or 4.3 $\times 10^{9} d_{19}^{2} M_{\odot}$, where $d_{19}$ is the distance to the galaxy, assumed to be the same as that to the Virgo Cluster in units of $19 \mathrm{Mpc}$. This is equal, within the uncertainties, to the flux we attribute to NGC 4532 in our Arecibo mapping (see Table 4; the fluxes reported in HLS + 93 for NGC 4532/ DDO 137 did not properly correct for beam overlapsplease note that this error affected only the map of NGC

TABLE 4

COMPARISON OF RESULTS

\begin{tabular}{|c|c|c|c|c|c|c|c|c|}
\hline \multirow[b]{2}{*}{$\begin{array}{c}\text { GALAXY } \\
\text { (1) }\end{array}$} & \multicolumn{2}{|c|}{$M_{\mathrm{HI}}$} & \multicolumn{2}{|c|}{$V_{\text {sys }}$} & \multicolumn{2}{|c|}{$\Delta V_{50}$} & \multirow{2}{*}{$\begin{array}{c}\alpha_{\mathrm{H}_{\mathrm{I}}} \\
\mathrm{VLA}^{\mathrm{e}} \\
(8)\end{array}$} & \multirow{2}{*}{$\begin{array}{c}D_{\mathrm{HI}_{\mathrm{I}}} \\
\mathrm{AO}^{\mathrm{f}} \\
(9)\end{array}$} \\
\hline & $\begin{array}{l}\mathrm{VLA}^{\mathrm{a}} \\
(2)\end{array}$ & $\begin{array}{c}\mathrm{AO}^{\mathrm{b}} \\
(3)\end{array}$ & $\begin{array}{l}\mathrm{VLA}^{\mathrm{c}} \\
(4)\end{array}$ & $\begin{array}{c}\mathrm{AO}^{\mathrm{c}} \\
(5)\end{array}$ & $\begin{array}{l}\mathrm{VLA}^{\mathrm{d}} \\
(6)\end{array}$ & $\begin{array}{l}\mathrm{AO}^{\mathrm{d}} \\
(7)\end{array}$ & & \\
\hline NGC $4532 \ldots . .$. & 43 & 39 & 2005 & 2024 & 161 & 191 & 5.3 & 66 \\
\hline DDO $137 \ldots \ldots$. & 8.6 & 16 & 2057 & 2044 & 70 & 124 & 3.2 & 94 \\
\hline Cloud A ........ & 1.3 & 1.1 & 1941 & 1948 & 33 & 39 & $\ldots$ & $\ldots$ \\
\hline Cloud B ........ & 3.9 & $\ldots$ & 2020 & 2020 & 91 & 87 & $\ldots$ & $\ldots$ \\
\hline Cloud C ........ & 2.6 & 3.0 & 1886 & 1879 & 76 & 82 & $\ldots$ & $\ldots$ \\
\hline Group ........... & 76 & 83 & 1999 & 2012 & 165 & 164 & $\ldots$ & 149 \\
\hline
\end{tabular}

${ }^{a} \mathrm{H}$ I mass in units of $10^{8} d_{19}^{2} M_{\odot}$.

b Same units as col. (1); these values supercede those in HLS + 93.

c Systemic velocities in units of $\mathrm{km} \mathrm{s}^{-1}$.

${ }^{d}$ Profile widths at $50 \%$ in $\mathrm{km} \mathrm{s}^{-1}$.

${ }^{e} \mathrm{H}$ i scale length in kiloparsecs.

${ }^{\mathrm{f}} \mathrm{H}$ I maximum diameter in kiloparsecs. 


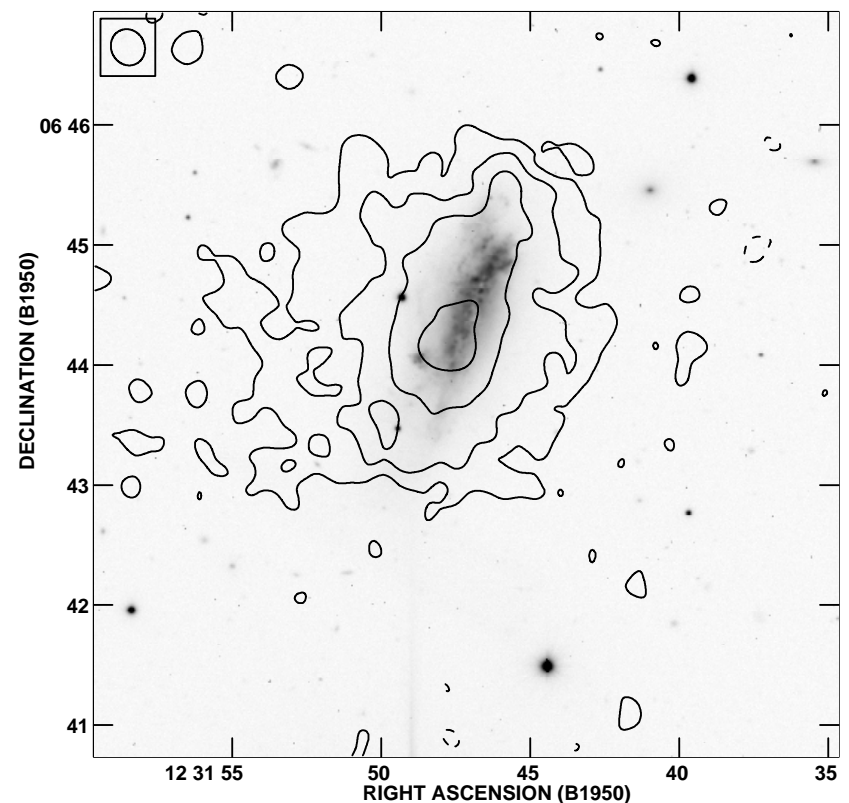

FIG. $4 a$

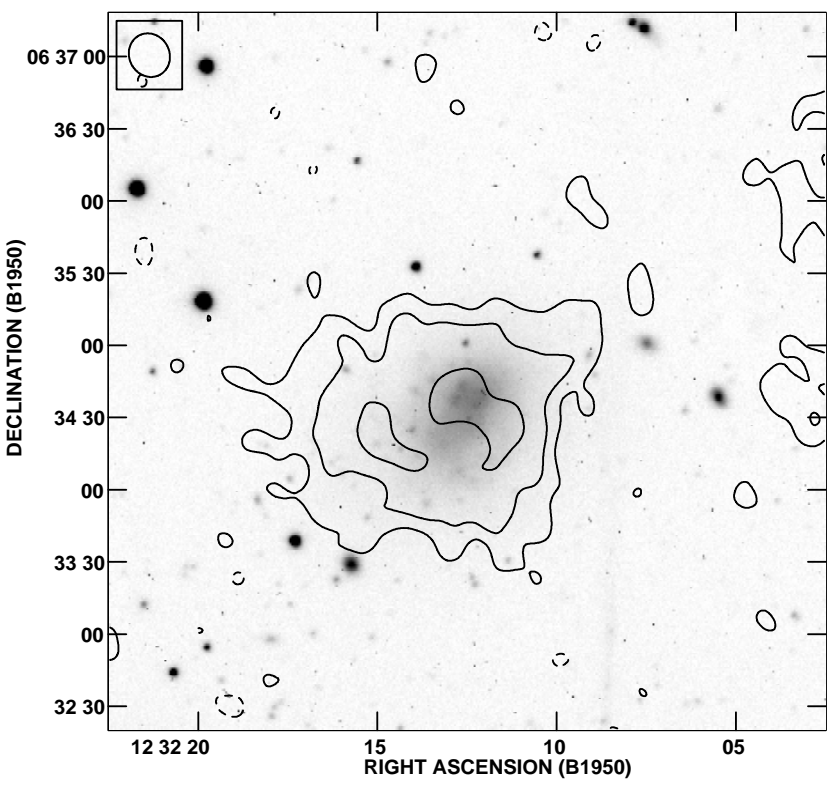

Fig. $4 b$

FIG. 4. - Contours of total hydrogen emission from (a) NGC 4532 and (b) DDO 137 integrated over all line-bearing channels from our combined C + D cube superposed on $R$-band CCD images from Lu et al. (1998). Neutral hydrogen contour levels are $-7.2,7.2,14.3,28.6$, and $57.2 \times 10^{20}$ atoms $\mathrm{cm}^{-2}$ for NGC 4532 and $-3.6,3.6,7.2$, and $14.3 \times 10^{20}$ atoms cm ${ }^{-2}$ for DDO 137. The CCD images employ a logarithmic transfer function, with the stretch chosen to show faint outer features without completely saturating the central parts of the galaxy.

4532/DDO 137, not the other maps reported in HLS + 93). The $\mathrm{H}$ I distribution is displaced from the optical image of the galaxy in the direction of DDO 137, and there is a flaring of $\mathrm{H}$ I toward the east with a distinct tail of $\mathrm{H} \mathrm{I}$ extending from the south end of the galaxy toward the east. The velocity field is similarly distorted; at the north end the contours are centered on the optical major axis and are consistent with a rising rotation curve symmetric about the optical center. But to the south the contours are skewed, probably by tidal interaction, and the flaring $\mathrm{H}$ i toward the east evidently is kinematically distinct from the rest of the $\mathrm{H}$ I in the galaxy.

Although the observed velocity field does not constrain the position angle or inclination of the innermost rings of

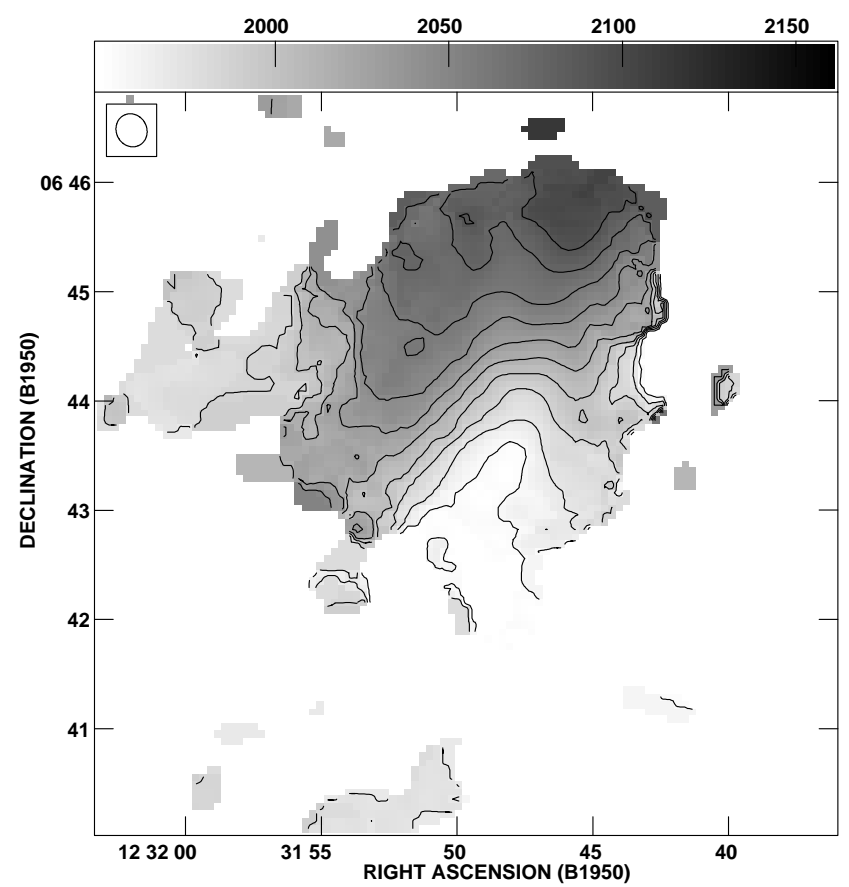

FIG. $5 a$

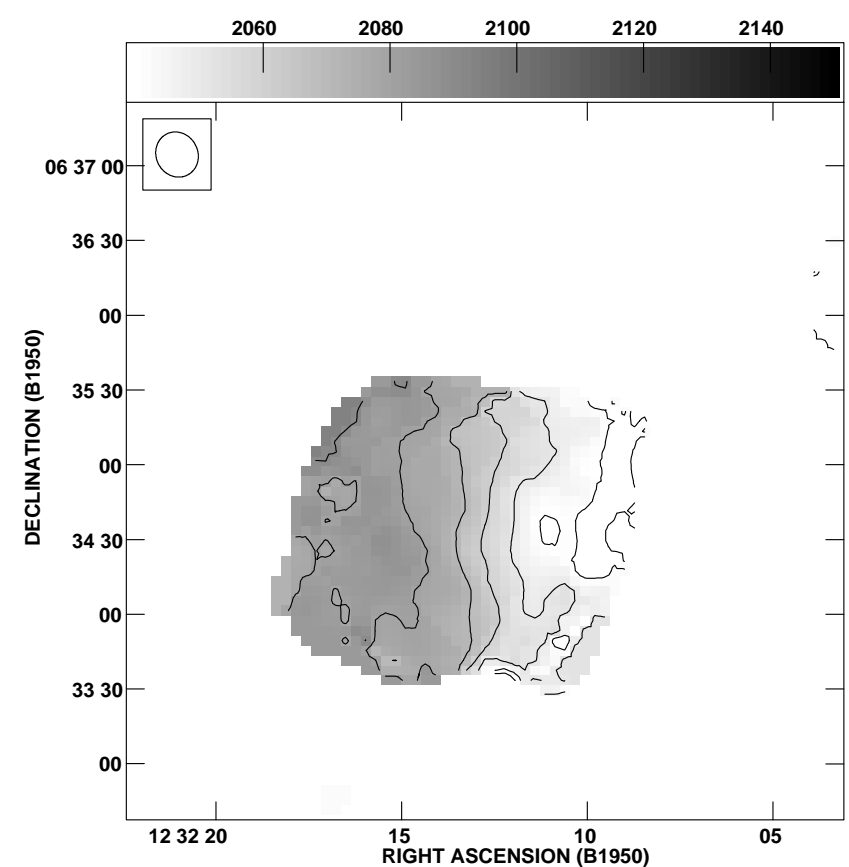

FIG. $5 b$

Fig. 5.-Isovelocity contours superposed on a gray-scale image of the velocity field from the merged C + D data of $(a)$ NGC 4532 and $(b)$ DDO 137. Contours are drawn from 1955 to 2090 in steps of $15 \mathrm{~km} \mathrm{~s}^{-1}$ for NGC 4532 and from 2030 to 2090 in steps of $10 \mathrm{~km} \mathrm{~s}^{-1}$ for DDO 137. 


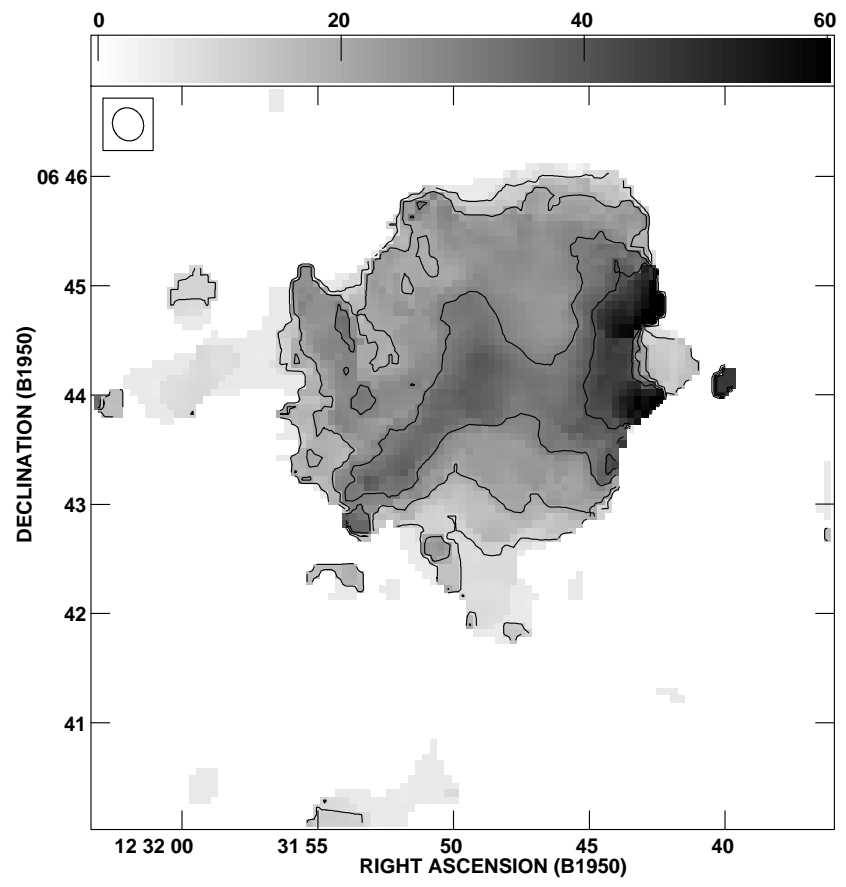

FIG. $6 a$

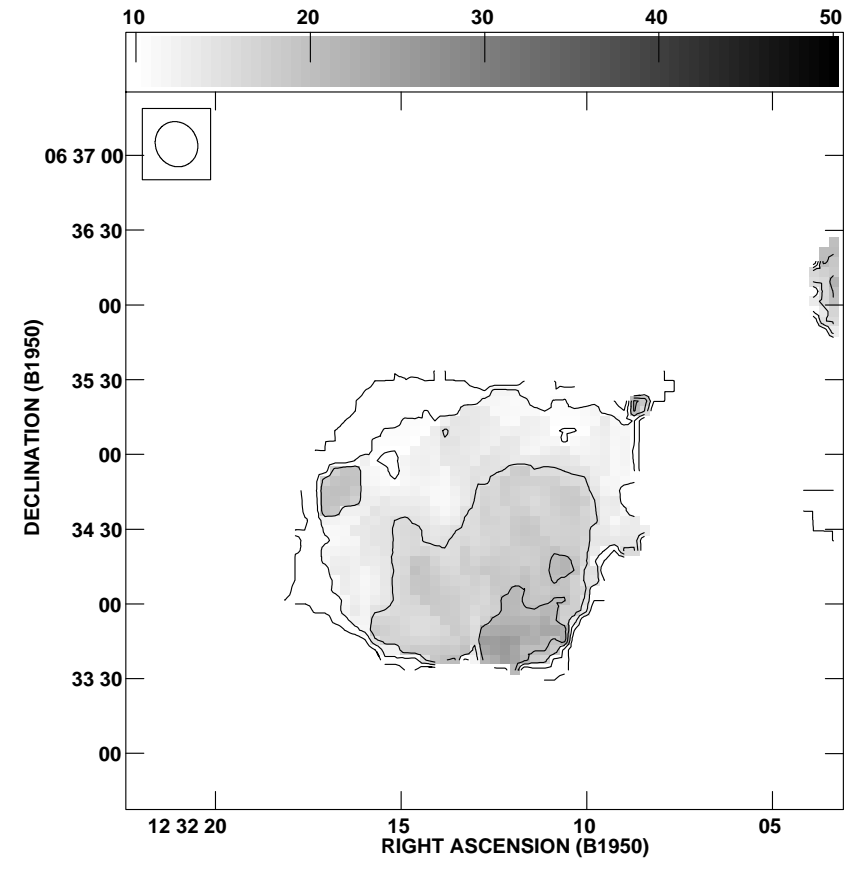

FIG. $6 b$

Fig. 6. - Second-moment contours superposed on a gray-scale image of the second-moment velocity field from the merged C + D data of $(a)$ NGC 4532 and (b) DDO 137. Contours are drawn from 10 to 40 in steps of $10 \mathrm{~km} \mathrm{~s}^{-1}$ for NGC 4532 and from 5 to 20 in steps of $5 \mathrm{~km} \mathrm{~s}^{-1}$ for DDO 137.

NGC 4532, we do obtain convergence, at values close to those determined optically, for the annulus having inner radius $50^{\prime \prime}$ and outer radius $70^{\prime \prime}$. Consequently, we adopt those values: position angle of the receding end of the major axis $347^{\circ}$, inclination $72^{\circ}$, and systemic velocity $2005 \mathrm{~km}$ $\mathrm{s}^{-1}$, for all rings of the galaxy. The galaxy's center is not well determined by the optical data, due to an apparent very strong off-center starburst, and the outer portions of the $\mathrm{H}$ I distribution and its velocity field are evidently perturbed by tidal interactions within the system. The central part of the galaxy's velocity field should be least affected by external influences, so the systemic velocity was chosen to make the central part of the rotation curve symmetric. The

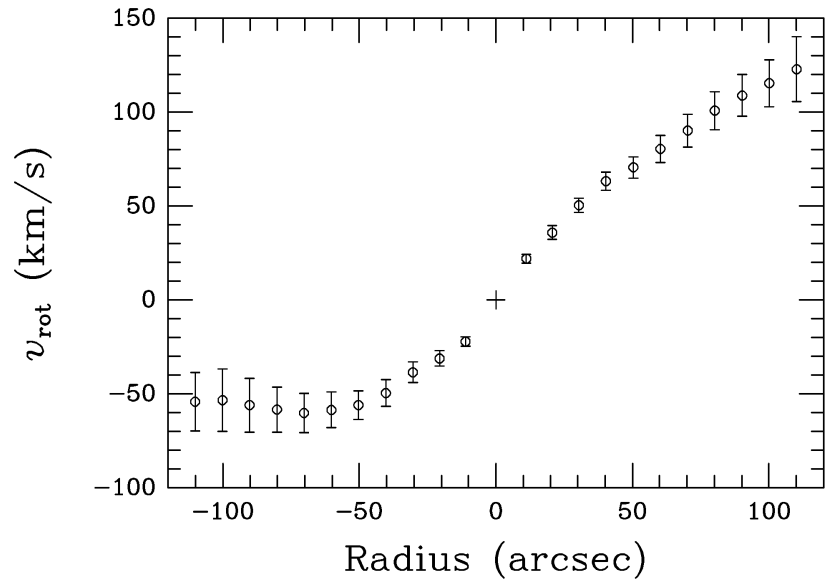

FIG. $7 a$ resulting rotation curve is displayed in Figure 7a. It appears to flatten on the approaching (southeast) end but continues to rise in more-or-less solid-body fashion on the receding end of the major axis. The maximum "rotation" speed observed is $123 \mathrm{~km} \mathrm{~s}^{-1}$ on the receding side of the galaxy; the approaching side appears to level off at a velocity of about $58 \mathrm{~km} \mathrm{~s}^{-1}$. If we average the rotation velocities within entire rings, as shown in Figure $10 a$ below, the maximum rotation speed is $102 \mathrm{~km} \mathrm{~s}^{-1}$ at a radius of $100^{\prime \prime}=9.2 \mathrm{kpc}$. The second-moment map indicates a rather uniform velocity dispersion within the $\mathrm{C}+\mathrm{D}$ array beam, around $20 \mathrm{~km} \mathrm{~s}^{-1}$ over most of the map, decreasing to 10 $\mathrm{km} \mathrm{s}^{-1}$ at the outskirts and with one large off-center region

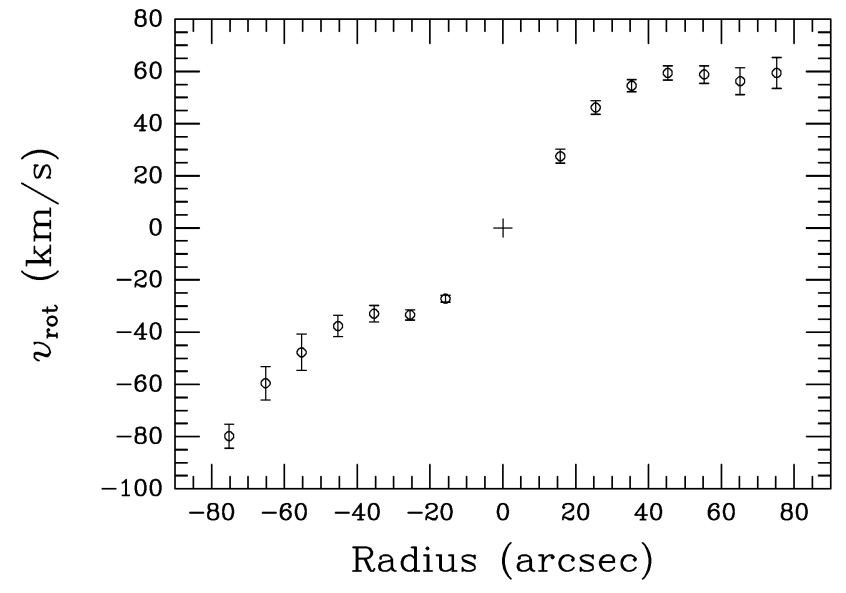

FIG. $7 b$

FIG. 7. - Rotation curves for (a) NGC 4532 and (b) DDO 137 determined by fitting the velocity fields within consecutive annuli. For NGC 4532 , the center position, systemic velocity $\left(2005 \mathrm{~km} \mathrm{~s}^{-1}\right)$, position angle, and inclination were held fixed for all rings at values discussed in the text. Positive velocities are receding and positive radii are toward the northwest. For DDO 137, the center position, systemic velocity $\left(2057 \mathrm{~km} \mathrm{~s}^{-1}\right)$, and inclination were held fixed for all rings at values discussed in the text; the position angle was determined by the fit. Positive velocities are receding and positive radii are toward the east. The cross in each panel marks the center in position and rotation velocity. 


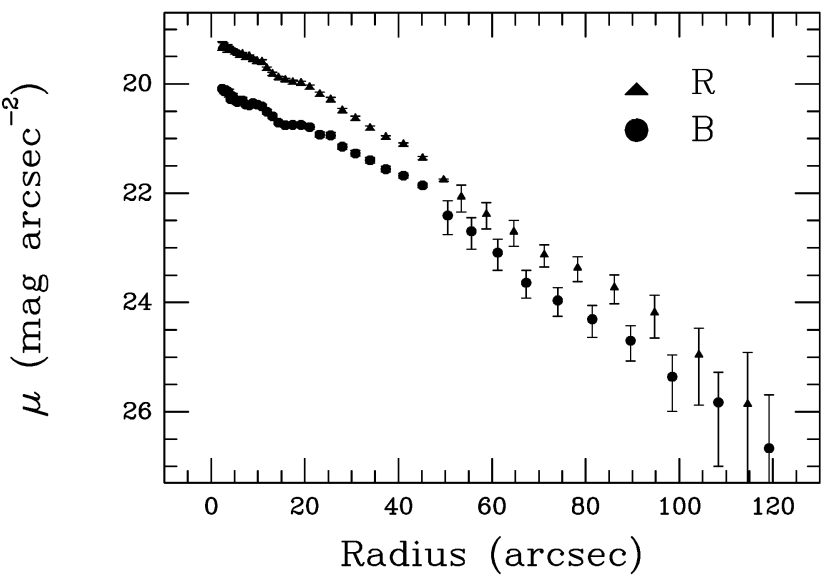

FIG. $8 a$

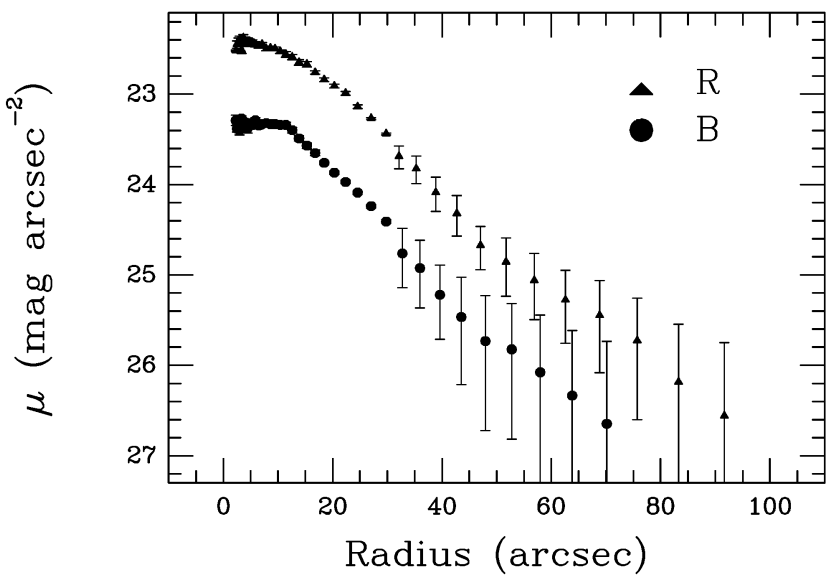

FIG. $8 b$

FIG. 8. - B (circles) and $R$ (triangles) surface brightness profiles for (a) NGC 4532 and (b) DDO 137 from Palomar CCD imaging

in which the dispersion rises above $30 \mathrm{~km} \mathrm{~s}^{-1}$. Since our synthesized beam is large compared with the scale on which the rotation curve rises, we expect the central part of the map to overestimate the dispersion. The dispersion is also likely to be overestimated around the outer edges of the map due to uneven blanking of the individual planes. The relevant dispersion for dynamical considerations is that in the outer regions; we adopt a value of $\sigma_{z}=24 \mathrm{~km} \mathrm{~s}^{-1}$, the average over the southeast and northwest portions of the second-moment field avoiding the center and avoiding the outer edges. This may still be a mild overestimate, but fortunately the dispersion is small compared to the rotation velocity so that errors in the dispersion have little effect on dynamical mass estimates. The dynamical mass $M_{\mathrm{dyn}}=$ $\left(v_{\mathrm{rot}}^{2}+3 \sigma_{z}^{2}\right) r / G$ is then $2.6 \times 10^{10} d_{19} M_{\odot}$.

The surface brightness profiles of NGC 4532 in $B$ and $R$, from LHS98, are shown in Figure $8 a$ with the resulting stellar and $\mathrm{H}$ I surface density profiles following in Figure $9 a$. Neither the gas mass surface density (including the contribution from primordial helium by multiplying by a factor of $4 / 3$ ) nor the stellar mass surface density (obtained from the $R$ surface brightness profile, assuming a constant $\left.M_{*} / L_{R}\right)$ is precisely exponential, but reasonable exponential fits can be made to each surface density distribution and to their sum. In Figure $10 a$, the dashed curve is the contribution to the rotation velocity from the gas, assuming it is in a thin disk. As is evident in Figure 4, the gas "disk" is far from thin and therefore produces a smaller rotation velocity for given $\mathrm{H}$ I mass than the infinitesimally thin disk assumed in Figure 10a. Furthermore, the off-center starburst causes the center of the gas disk not to coincide with the apparent center of the stellar disk. Instead of the usual "maximum-disk" construction, we define a "supermaximal stellar disk" to give a rotation velocity curve close to the observed curve at intermediate radii (slightly exceeding the observed curve at radii $\leq 40^{\prime \prime}$ to allow for beam smearing), without any contribution from gas or dark matter. This is intended to give the largest reasonable estimate of the stellar mass-to-light ratio, with the underestimate due to the thin disk assumption compensated by the overestimate due to the neglect of the gas. The rotation velocity resulting from this "supermaximal stellar disk" is shown as the solid curve in Figure $10 a$ and requires a mass-to-light ratio $M_{*} / L_{R}=0.24 M_{\odot} / L_{\odot}$ giving a total stellar mass $M_{*}=2.4$ $\times 10^{9} d_{19}^{2} M_{\odot}$. This is a small $M_{*} / L_{R}$ but may be consistent with the indications of a strong starburst in the optical images (Bruzual \& Charlot 1993). We will discuss this point further in LHS98. If the rotation curve averaged within

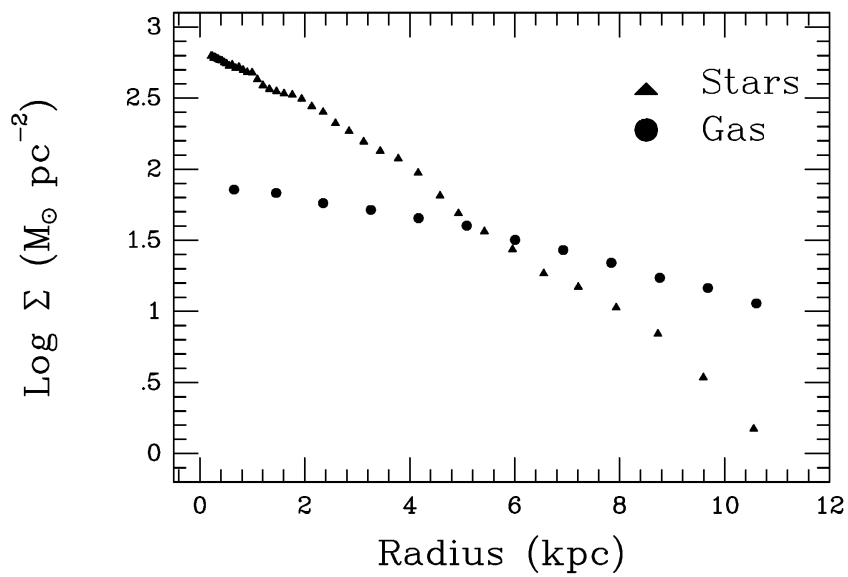

FIG. $9 a$

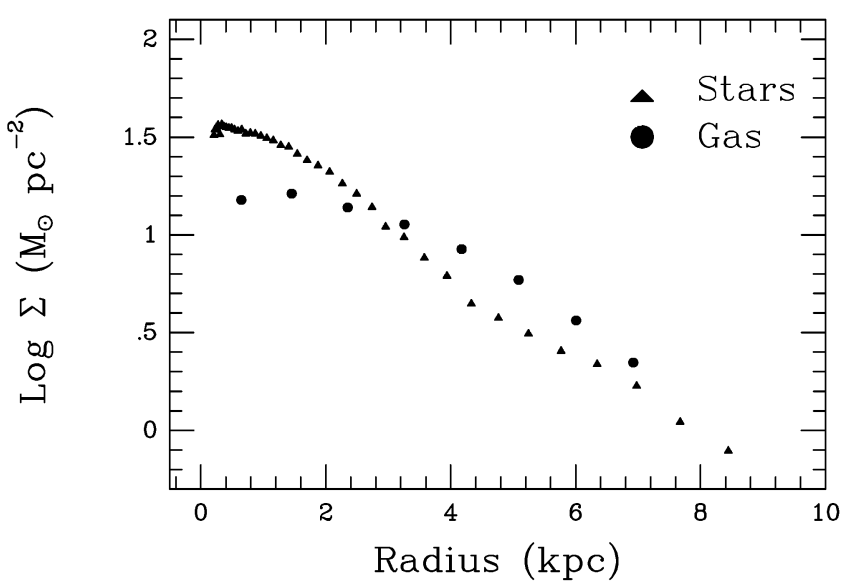

FIG. $9 b$

FIG. 9.- Mass surface densities in stars (triangles) and gas (circles) for (a) NGC 4532 and (b) DDO 137 . We have assumed $M_{*} / L_{R}=1 M_{\odot} / L_{\odot}$ for the purposes of these plots and have included neutral hydrogen and primordial helium gas but have neglected molecular and ionized gas. 


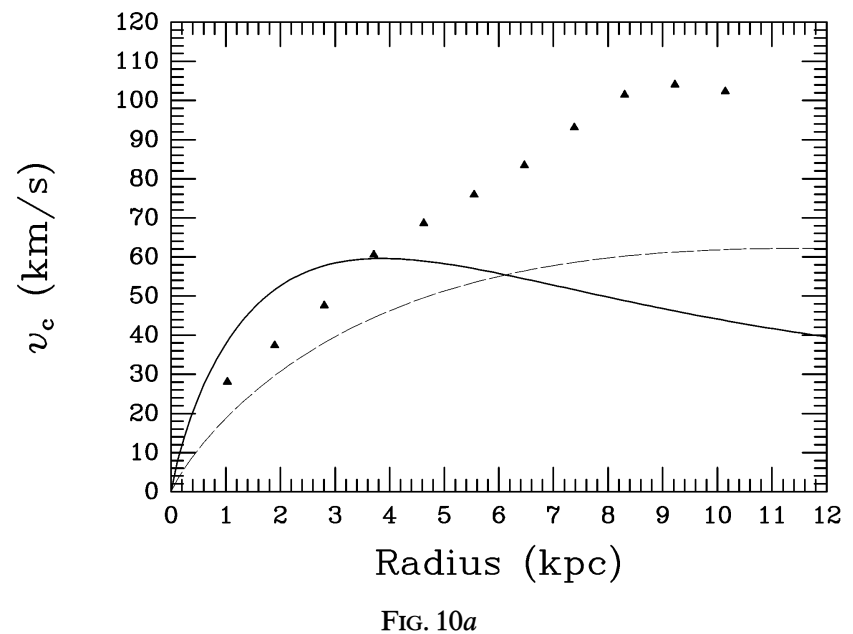

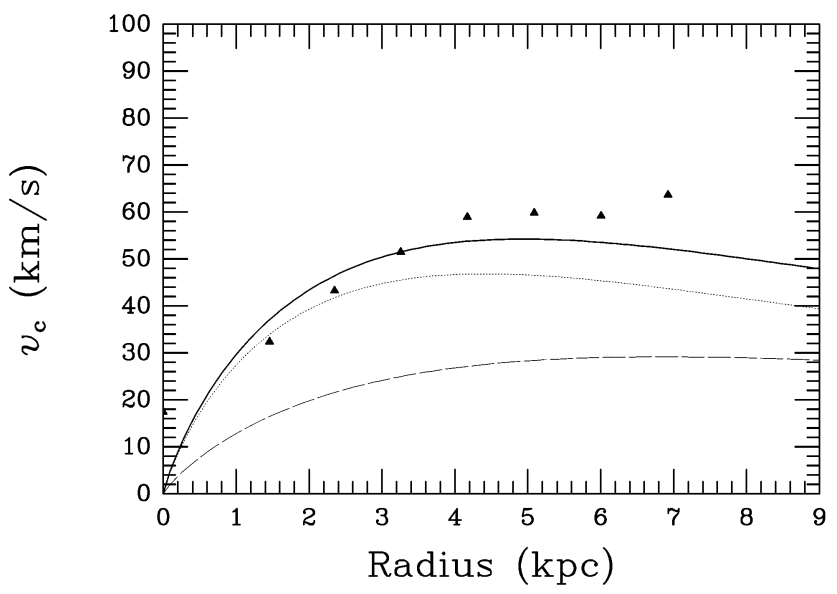

FIG. $10 b$

FIG. 10.- Rotation curves (triangles) of (a) NGC 4532 and (b) DDO 137 averaged over entire rings. The dashed curve in each panel shows the contribution of a thin exponential disk fitted to the gas, including neutral hydrogen and primordial helium. For NGC 4532 the solid curve shows the contribution of the "supermaximal" thin exponential disk fitted to the stars assuming $M_{*} / L_{R}=0.24$ (see text). For DDO 137 the dotted curve shows the contribution of a thin stellar exponential disk having $M_{*} / L_{R}=2.2 M_{\odot} / L_{\odot}$ while the solid curve shows that of a thin exponential disk fit to the sum of stars plus gas.

entire annuli of the galaxy is appropriate dynamically, we need a significant contribution from dark matter in the outer parts of the galaxy.

\section{2. $D D O 137$}

Corresponding maps of total $\mathrm{H} \mathrm{I}$ and velocity field for the SmIV galaxy DDO 137 from the merged C + D array data cube are displayed in Figures $4 b$ and $5 b$. The integrated emission in the merged C + D map is $10.1 \mathrm{Jy} \mathrm{km} \mathrm{s}{ }^{-1}$, or $8.6 \times 10^{8} d_{19}^{2} M_{\odot}$. We cannot make a comparison directly with the Arecibo map in this case, since the Arecibo mapping did not allow us to separate out DDO 137 from the plume extending west and curving south from the galaxy. There are weak indications in the $\mathrm{H}$ I map of a tidal streamer reaching toward the plume but no strong distortions of the velocity field. The relatively weak rotation of the galaxy appears approximately solid body in form over most of the first-moment map.

The position angle from elliptical fits to the outer isophotes of DDO 137 in LHS98 is $130^{\circ}$, but the kinematic major axis is apparently very close to east-west. Fitting the velocity field within consecutive annuli, with the inclination held fixed at $30^{\circ}$ since it is not well-constrained by the $\mathrm{H} \mathrm{I}$ data, indicates that the position angle of consecutive rings gradually shifts from about $96^{\circ}$ for inner rings to about $82^{\circ}$ for the outermost couple. To plot the rotation curve shown in Figure 7b, we held inclination, center position, and systemic velocity fixed for all rings but allowed the position angle to be determined by the fit. The rotation curve is probably best described as solid body with perturbations. The deviations from a solid-body curve should not be taken too seriously in model fitting, however, since the H I map indicates interaction effects. In particular, the outermost rings may be sampling portions of the extended plume rather than DDO 137 itself. The second-moment map gives an average velocity dispersion $\sigma_{z}=15 \mathrm{~km} \mathrm{~s}^{-1}$ within the $\mathrm{C}+\mathrm{D}$ array beam, avoiding the edges of the map, which may be corrupted by blanking effects; this is presumably an overestimate, since the beam is not small compared with the scale on which the rotation curve rises. We will use $\sigma_{z}=10$ $\mathrm{km} \mathrm{s}^{-1}$ for dynamical purposes. If we can take at face value the highest measured rotation speed, $59(0.5 / \mathrm{sin} i) \mathrm{km} \mathrm{s}^{-1}$, at $75^{\prime \prime} .2=6.9 \mathrm{kpc}$ on the receding side of the galaxy, farthest from the apparent tidal stream toward cloud $\mathrm{B}$, we find a dynamical mass $M_{\mathrm{dyn}}=5.6\left[(0.5 / \sin i)^{2}+0.086\left(\sigma_{z} / 10\right)^{2}\right]$ $\times 10^{9} d_{19} M_{\odot}$.

The surface brightness profiles in $B$ and $R$, from LHS98, and the $\mathrm{H}$ I surface density profile of DDO 137 are shown in Figure $8 b$ and $9 b$. The gas mass surface density includes the contribution from primordial helium, by multiplying by a factor of $4 / 3$, and the stellar mass surface density is obtained from the $R$ surface brightness profile, assuming a constant $M_{*} / L_{R}$. As for NGC 4532, reasonable exponential fits can be made to each surface density distribution and to their sum. In Figure $10 b$ we show the contributions of those exponential disks to the rotation curve of the galaxy, with $M_{*} / L_{R}$ adjusted to match the inner portion of the star-plusgas disk to the corresponding portion of the rotation curve (exceeding the innermost two points to allow for beam smearing and ignoring the thickness of the disks). In this case $M_{*} / L_{R}=2.2 M_{\odot} / L_{\odot}$ gives a reasonable fit to the entire rotation curve, except for the outermost point, which is probably affected by noncircular gas motions. There is perhaps no need for a dark matter halo in DDO 137; however, the maximum-disk value of $M_{*} / L_{R}$ is probably an overestimate if the considerations of Bottema (1997) outweigh the countereffect of the thickness of the gas and stellar disks.

\subsection{Three Gas Concentrations outside the Galaxies}

Cloud A, detected separately in the Arecibo spectra, the $\mathrm{D}$ array data and the $\mathrm{C}$ array data, is clearly visible in the total H I map about $6^{\prime}$ east of NGC 4532, $7^{\prime}$ due north of DDO 137. The total $\mathrm{H}$ I emission from the cloud, summed over the channels (1906-1958 $\left.\mathrm{km} \mathrm{s}^{-1}\right)$ that show line emission at that location, is shown in Figure 11a. It is resolved by our $\mathrm{C}+\mathrm{D}$ array data but is only a few beamwidths across. There is some indication of rotation (or tidal shear) in the velocity field, with the northwest end having lower velocity than the southeast end, but the end-to-end difference is only about two of our velocity channels and the contours are far from regular in shape. 


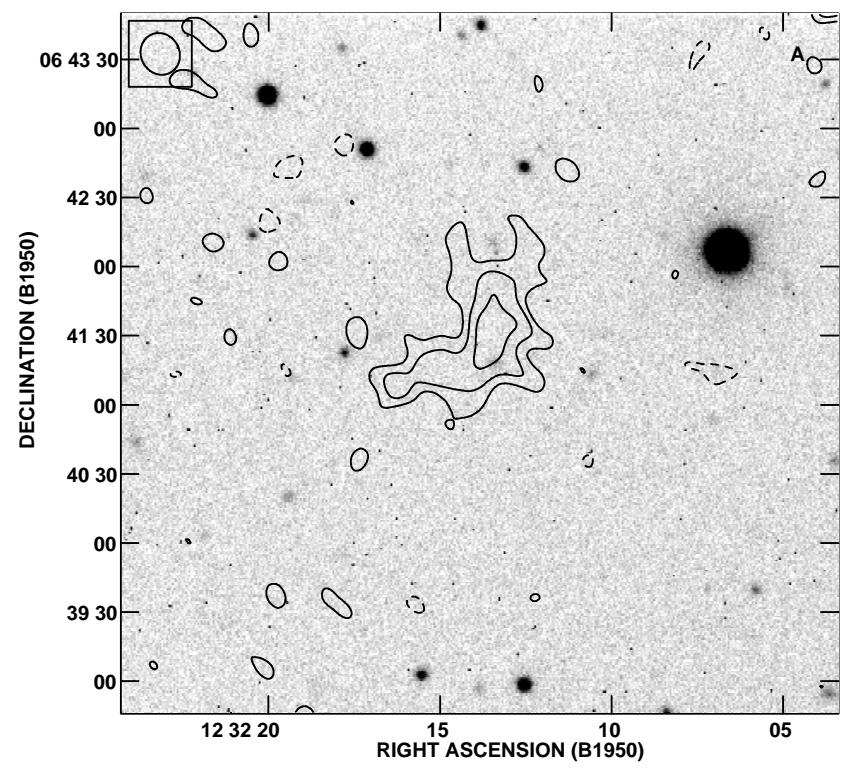

FIG. $11 a$

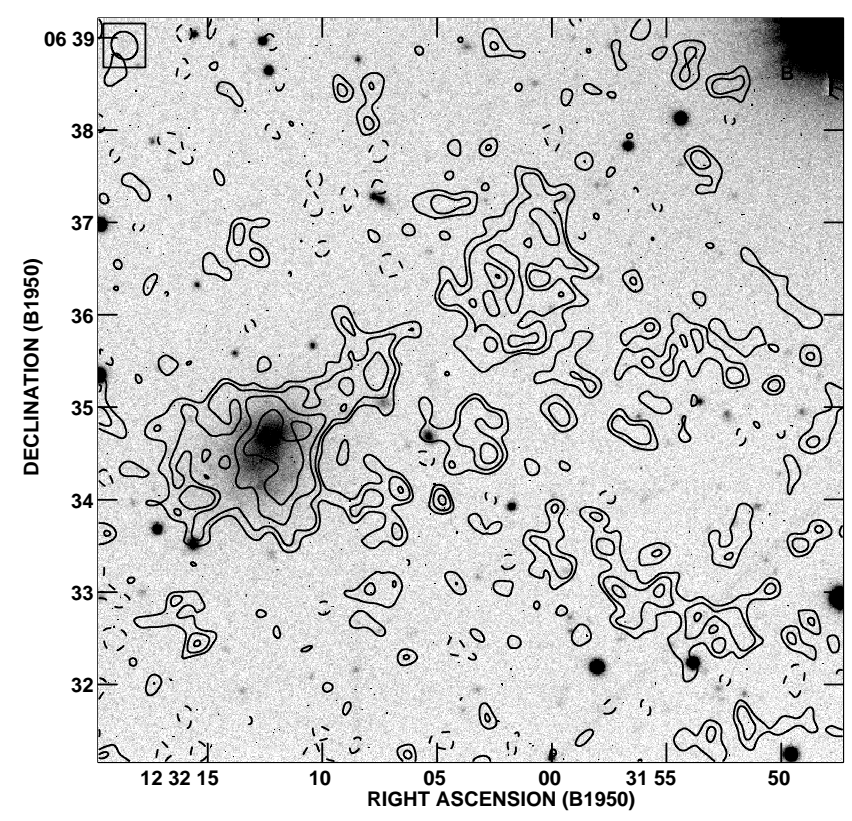

FIG. $11 b$

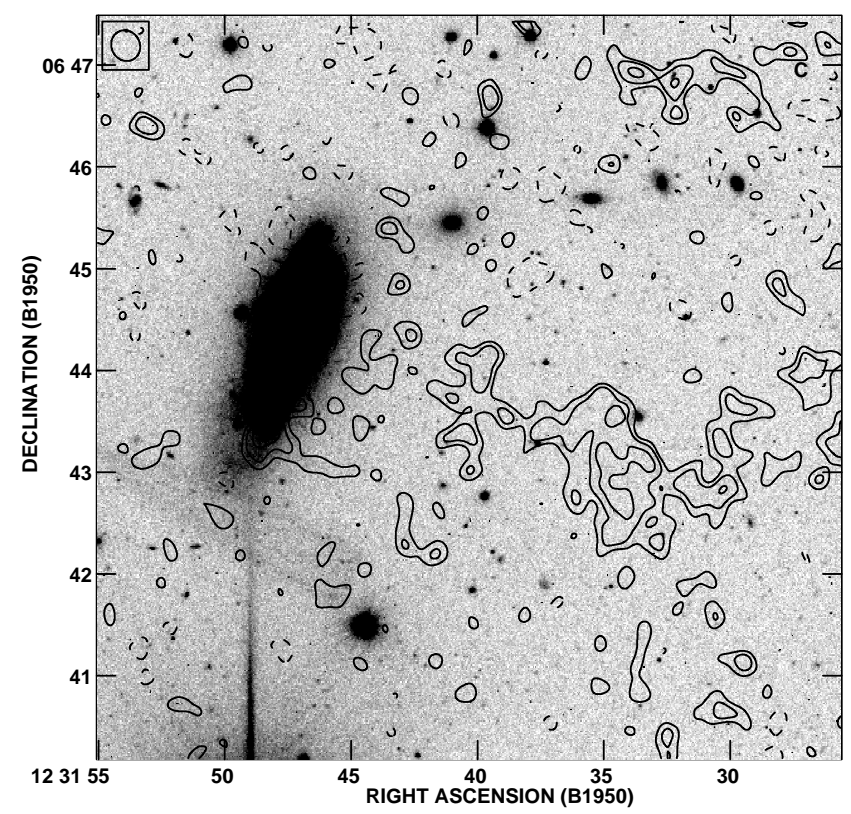

FIG. 11c

FIG. 11.-Contours of total hydrogen emission from clouds $(a) \mathrm{A},(b) \mathrm{B}$, and $(c) \mathrm{C}$, integrated over all line-bearing channels from our C $+\mathrm{D}$ array combination (1906-1958 $\mathrm{km} \mathrm{s}^{-1}$ for A, 1906-1990 km s $\mathrm{km}^{-1}$ for B, and $1823-1927 \mathrm{~km} \mathrm{~s}^{-1}$ for C). The contours are overlaid on gray-scale images of $B$-band CCD frames from LHS98. Contour levels are $-2.1,2.1,3.2$, and $4.8 \times 10^{20}$ atoms cm ${ }^{-2}$ for A, $-3.6,3.6,5.4,8.0$, and $12.1 \times 10^{20}$ atoms cm ${ }^{-2}$ for B, and $-2.9,2.9,4.3$, and $6.4 \times 10^{20}$ atoms cm ${ }^{-2}$ for $\mathrm{C}$. The stretch in each logarithmic $B$-band image is chosen to bring out the faintest possible features. Traces of DDO 137 and NGC 4532 remain near the left-hand edges of the frames for B and C, respectively. The triangular "paper-clip" artifact just south of NGC 4532 in the image for cloud $\mathrm{C}$ is spurious.

Cloud $\mathrm{B}$ is a concentration within the extended plume that extends west, then curves south, from DDO 137 in our Arecibo map (HLS + 93). It is visible as a distinct cloud in the $\mathrm{D}$ array and $\mathrm{C}$ array data separately, in the velocity range $1906-1990 \mathrm{~km} \mathrm{~s}^{-1}$. The total $\mathrm{H}_{\mathrm{I}}$ map is shown in Figure $11 b$; it is clearly resolved by our $\mathrm{C}+\mathrm{D}$ array data. The velocity field does not display any regular pattern, although there are larger velocity gradients within cloud B than within cloud $\mathrm{A}$.

Cloud C (Fig. 11c) appears at lower velocity than any other feature in the maps, in the range $1823-1927 \mathrm{~km} \mathrm{~s}^{-1}$ channels. It is visible in the Arecibo data, as well as in the C and $\mathrm{D}$ array data separately. As with the other two clouds, there is no clear order to its velocity field.

\section{DISCUSSION}

\subsection{Comparisons with Arecibo Spectra}

Table 4 compares measured quantities from the VLA mapping reported here with corresponding quantities from our Arecibo mapping reported in HLS + 93. Unfortunately, the integrated $\mathrm{H}_{\mathrm{I}}$ fluxes and hydrogen masses 
given in Table 2 of HLS +93 for the components of the NGC 4532/DDO 137 system did not correctly account for beam overlaps; the values cited in Table 4 of this paper supersede those reported earlier. (This error in HLS + 93 affected only NGC 4532/DDO 137, not the other galaxies discussed there.) With the heavily weighted zero-spacing fluxes used here, the VLA integrated $\mathrm{H}$ I fluxes for NGC 4532 agrees well with the $\mathrm{H}$ I emission integrated over the Arecibo spectra attributed to that galaxy. For DDO 137, the Arecibo spatial resolution was not adequate to separate the galaxy from the extended plume, which contains cloud $\mathrm{B}$, so direct comparison of total fluxes is not meaningful.

The integrated spectra from the merged D + C array data cube also agree quite well in detail with the singlebeam Arecibo spectra acquired when Arecibo was pointed at the optical centers of the two galaxies. Figure $12 \mathrm{com}$ pares the spectra for each of the galaxies. Clearly, that measurements of systemic velocity and profile widths made from these spectra would agree within a fraction of the VLA channel spacing as shown in Table 4. For NGC 4532, onehalf the Arecibo profile width at $50 \%$ of the nearest peak $\left(80.5 \mathrm{~km} \mathrm{~s}^{-1}\right)$ is a bit smaller than the maximum rotation velocity inferred from the VLA map $\left(102 \sin 72^{\circ}=97 \mathrm{~km}\right.$ $\mathrm{s}^{-1}$ ). The inferred systemic velocities do not agree quite so well: $2020 \mathrm{~km} \mathrm{~s}^{-1}$ from the midpoint of the two $50 \%$ points of the Arecibo profile compared with $2005 \mathrm{~km} \mathrm{~s}^{-1}$ from fitting the velocity field in the VLA map. For DDO 137, one-half the Arecibo profile width at $50 \%$ of peak is $33.4 \mathrm{~km}$ $\mathrm{s}^{-1}$, while the maximum rotation velocity from the VLA map gives $59 \sin 30^{\circ}=30 \mathrm{~km} \mathrm{~s}^{-1}$. The systemic velocity taken to be the midpoint of the points at which the Arecibo profile falls to $50 \%$ of its peak is $2066 \mathrm{~km} \mathrm{~s}^{-1}$, slightly larger than the value reported for DDO 137 plus plume in Table 4; we inferred a value of $2057 \mathrm{~km} \mathrm{~s}^{-1}$ from the VLA velocity field.

Integrated spectra for each of the three gas concentrations are compared with the Arecibo spectra from the closest pointings in Figure 13. The integration in each case extends over the largest contiguous contour in Figures 11a, $11 b$, or $11 c$. Here the agreement is not so good, because the Arecibo beam is not pointed at the center of the cloud in each case and has collected flux from other parts of the plume or from the outskirts of the galaxies. In the case of cloud A, the bump in the Arecibo spectrum extending up to $2100 \mathrm{~km} \mathrm{~s}^{-1}$ is apparently due to incompletely subtracted sidelobes of NGC 4532. For cloud B, confusion with DDO 137 and the extended plume makes it impossible for us to separate out an $\mathrm{H}$ I mass for the cloud alone from Arecibo spectra, but for clouds $\mathrm{A}$ and $\mathrm{C}$ we can interpolate among the Arecibo spectrum nearest to each cloud's position and the six nearest neighbor beam positions (with some judgment by eye of what portion of each spectrum to attribute to the cloud). The resulting estimated $\mathrm{H}$ I masses, with uncertainties greater than $20 \%$, are displayed in Table 4 The integrated profile of each component and their sum are compared with the global Arecibo profile in Figure 14.

\subsection{Indicative Masses of the Three Gas Concentrations}

The extreme velocities encountered within cloud A are 1927 and $1952 \mathrm{~km} \mathrm{~s}^{-1}$, at opposite ends of the cloud separated by about $72^{\prime \prime}=6.6 d_{19} \mathrm{kpc}$. This suggests rotation, but the isovelocity contours are far from regular. The second-moment map has an average velocity dispersion $\sigma_{z}=7.5 \mathrm{~km} \mathrm{~s}^{-1}$, an overestimate since there is a velocity gradient on a scale comparable to the beam size. If we could assume that the cloud is in simple rotation and that our perspective is edge-on-neither assumption is likely to be true-we would have a dynamical mass $M_{\mathrm{dyn}}=$ $\left(v_{\text {rot }}^{2}+3 \sigma_{z}^{2}\right) r / G=2.5 \times 10^{8} d_{19} M_{\odot}$. However, the assumption of rotation is suspect; tidal shear is equally likely to be the cause of the velocity gradient. We consequently report in Table 5 only the indicative gravitational mass $M_{\text {ind }}=$ $3\left(\Delta V_{50} / 2\right)^{2} r / G=6.2 \times 10^{8} d_{19} \quad M_{\odot}$ (which would be the dynamical mass if $\Delta V_{50} / 2$ were a good measure of the lineof-sight component of the turbulent velocity within the cloud and if the cloud were in virial equilibrium). The integrated $\mathrm{H}$ I amounts to $1.5 \mathrm{Jy} \mathrm{km} \mathrm{s}^{-1}$, or $1.3 \times 10^{8} d_{19}^{2} M_{\odot}$, so the $\mathrm{H}$ I mass alone is probably not sufficient to bind the cloud if the virial theorem estimate approximates its true mass.

For cloud B, which we take to be the largest distinct clump near the center of Figure $11 b$, the situation is even less clear. The extreme velocities are approximately 1980 and $2050 \mathrm{~km} \mathrm{~s}^{-1}$, but the low velocity occurs in the center of the cloud. It would be incorrect to associate this velocity field with circular rotation. The width of the integrated

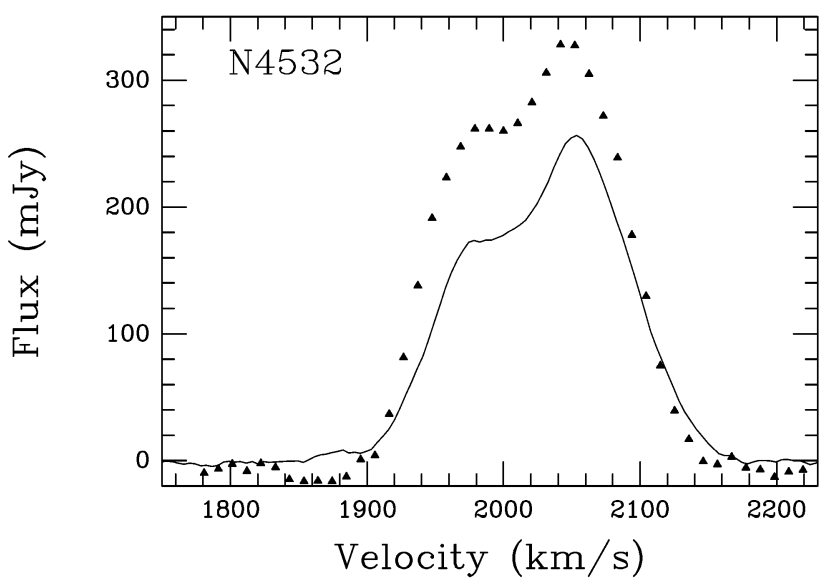

FIg. $12 a$

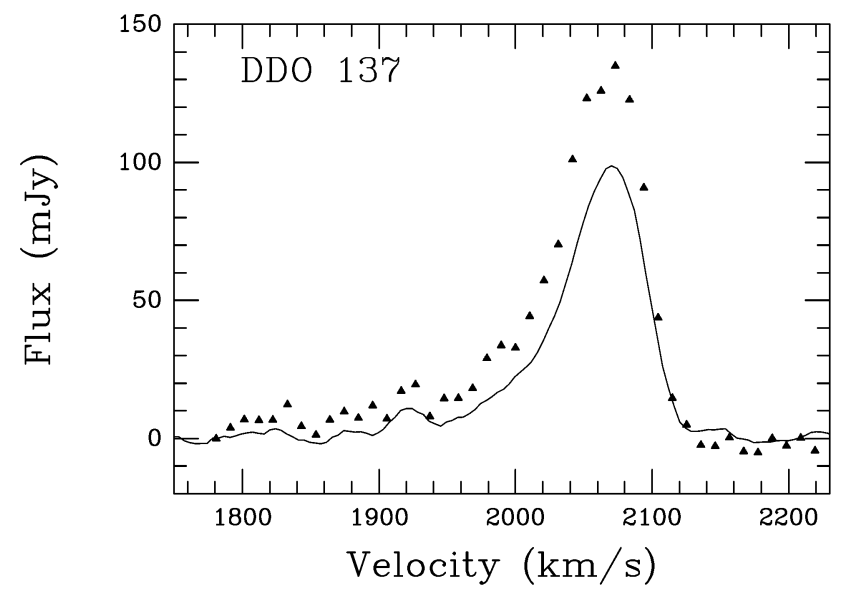

Fig. $12 b$

FIG. 12. - Spectra integrated over the individual galaxies (triangles) compared with the Arecibo central beam profile (solid line) for (a) NGC 4532 and (b) DDO 137. 


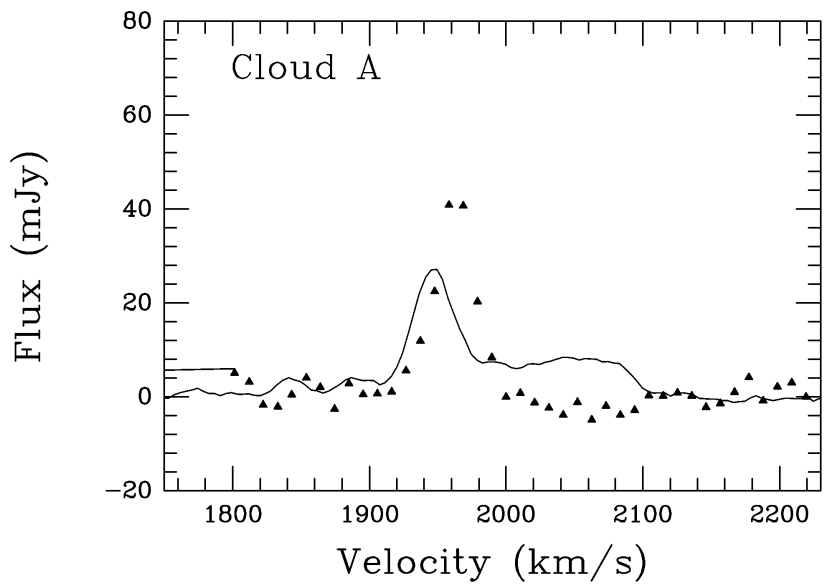

FIG. $13 a$

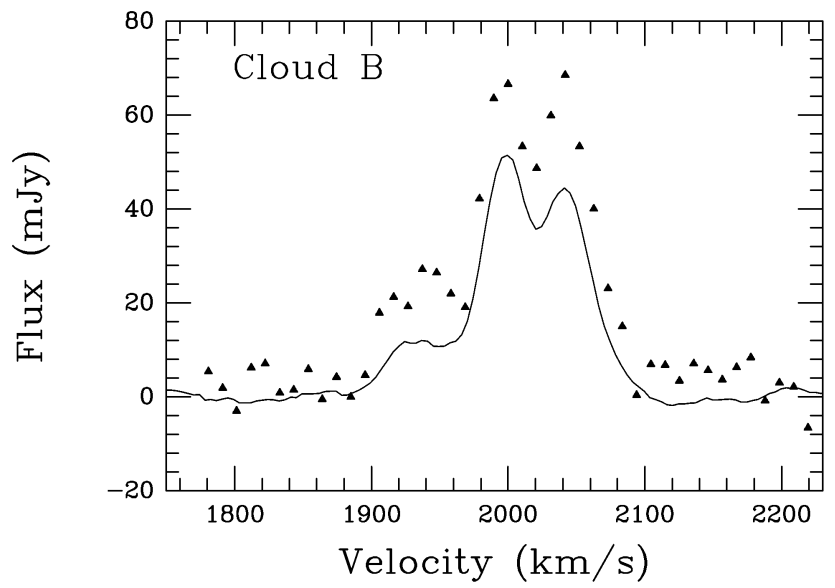

Fig. $13 b$

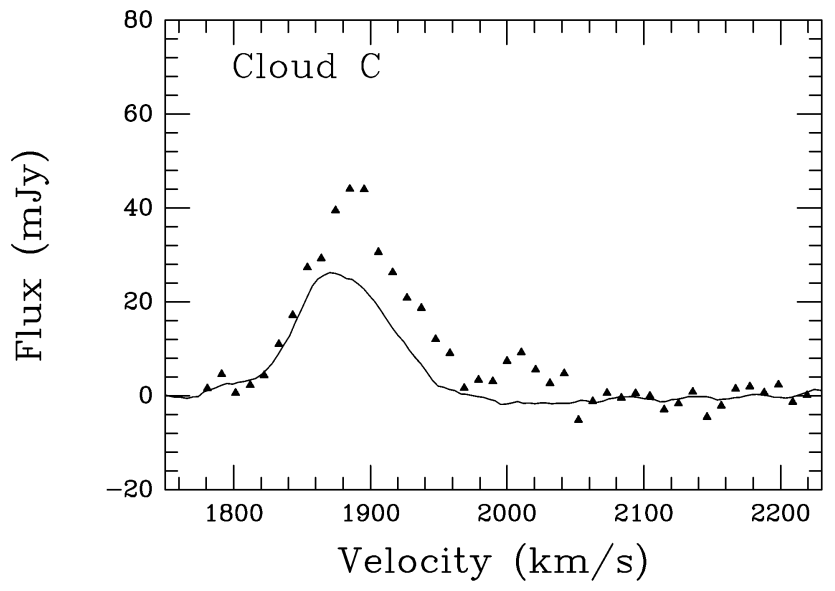

FIG. 13c

Fig. 13. - Spectra integrated separately (triangles) over clouds $(a) \mathrm{A},(b) \mathrm{B}$, and (c) C compared with the Arecibo single-beam profile (solid line) from the beam position closest to each cloud.

spectrum, Figure $13 b$, is $91 \mathrm{~km} \mathrm{~s}^{-1}$ at the points $50 \%$ down from the peaks of the double-horned profile. The velocity dispersion, from the average over the second-moment map in regions in which the velocity field is quiet, is $\sigma_{z}=19 \mathrm{~km}$ $\mathrm{s}^{-1}$, but that overestimates the local gas velocity dispersion as discussed above. The linear dimension of the cloud is $113^{\prime \prime}$, or $10.4 d_{19} \mathrm{kpc}$. The indicative gravitational mass is then $M_{\text {ind }}=3\left(\Delta V_{50} / 2\right)^{2} r / G=7.5 \times 10^{9} d_{19} M_{\odot}$. The inte- grated $\mathrm{H}$ I mass is $4.5 \mathrm{Jy} \mathrm{km} \mathrm{s}{ }^{-1}$, or $3.9 \times 10^{8} d_{19}^{2} M_{\odot}$. If cloud B is gravitationally bound, evidently much dark matter is required. But the assumption that it is in virial equilibrium is highly suspect, since the cloud lies in an extended plume.

Cloud C likewise does not have a well-ordered velocity field. Within the cloud itself, velocities range from about 1854 to $1894 \mathrm{~km} \mathrm{~s}^{-1}$, but in this case it is the high velocities

TABLE 5

DyNAMiCAL MasSes

\begin{tabular}{|c|c|c|c|c|c|c|}
\hline $\begin{array}{l}\text { Galaxy } \\
\text { (1) }\end{array}$ & $\begin{array}{c}v_{\text {rot }} \\
\left(\mathrm{km} \mathrm{s}^{-1}\right) \\
(2)\end{array}$ & $\begin{array}{c}\sigma_{z} \\
\left(\mathrm{~km} \mathrm{~s}^{-1}\right)\end{array}$ & $\begin{array}{c}r \\
\left(d_{19} \mathrm{kpc}\right) \\
(4)\end{array}$ & $\begin{array}{c}M_{\mathrm{dyn}} \\
\left(10^{8} d_{19} M_{\odot}\right) \\
(5)\end{array}$ & $\begin{array}{c}M_{\star} \\
\left(10^{8} d_{19}^{2} M_{\odot}\right) \\
(6)\end{array}$ & $\begin{array}{c}M_{\text {gas }} \\
\left(10^{8} d_{19}^{2} M_{\odot}\right) \\
(7)\end{array}$ \\
\hline NGC $4532 \ldots \ldots \ldots \ldots \ldots$ & 102 & $24^{\mathrm{a}}$ & 8.3 & 260 & $24(100)^{b}$ & 57 \\
\hline DDO $137 \ldots \ldots \ldots \ldots \ldots$ & 59 & $15^{\mathrm{a}}$ & 6.9 & $61^{\mathrm{c}}$ & $18(8)^{b}$ & 11.5 \\
\hline Cloud A ................... & $\ldots$ & $16^{\mathrm{d}}$ & 3.3 & $6.2^{\mathrm{e}}$ & $\ldots$ & 1.7 \\
\hline Cloud B .................. & $\ldots$ & $46^{\mathrm{d}}$ & 5.2 & $75^{\mathrm{e}}$ & $\ldots$ & 5.2 \\
\hline Cloud C $\ldots \ldots \ldots \ldots \ldots \ldots$ & $\ldots$ & $38^{\mathrm{d}}$ & 5.8 & $58^{\mathrm{e}}$ & $\ldots$ & 3.5 \\
\hline Group $(20 \%$ width $) \ldots \ldots$ & $\ldots$ & 141 & 74 & 2500 & $42(108)^{b}$ & 111 \\
\hline Group (virial) ............ & $\ldots$ & $\ldots$ & $\ldots$ & 4300 & $42(108)^{b}$ & 111 \\
\hline
\end{tabular}

${ }^{\text {a }}$ Overestimate from second-moment map; see text.

${ }^{b}$ Value using $M_{*} / L_{R}$ from rotation curve fit (value using $M_{*} / L_{R}=1$ ).

c $5.6\left[(0.5 / \sin i)^{2}+0.086\left(\sigma_{z} / 10\right)^{2}\right]$; see text.

${ }^{\mathrm{d}} \Delta V_{50} / 2$.

${ }^{\mathrm{e}}$ Indicative gravitational mass $3 \Delta V_{50}^{2} r / G$. 
that occur near the center of the cloud. The profile width at $50 \%$ of the peak of the spectrum, Figure $13 c$, is $76 \mathrm{~km} \mathrm{~s}^{-1}$. The beam scale line-of-sight dispersion $\sigma_{z}$ is about $17 \mathrm{~km}$ $\mathrm{s}^{-1}$, averaged over the second-moment map in regions in which the gradient in the first moment is small. The linear size of the cloud is $126^{\prime \prime}=11.6 d_{19} \mathrm{kpc}$. Taking one-half the profile width to indicate turbulent velocity as for clouds A and $\mathrm{B}$, above, we obtain an estimate of the indicative gravitational mass $M_{\text {ind }}=3\left(\Delta V_{50} / 2\right)^{2} r / G=5.8 \times 10^{9} d_{19} M_{\odot}$. The integrated $\mathrm{H} \mathrm{I}$ mass is $3.1 \mathrm{Jy} \mathrm{km} \mathrm{s}{ }^{-1}$, or $2.6 \times 10^{8} d_{19}^{2}$ $M_{\odot}$. The dark matter requirements are about as extreme as for cloud B, if cloud C is to be bound, but again the assumption of virial equilibrium is suspect since cloud $\mathrm{C}$ also lies within an extended stream.

The $\mathrm{H}$ I masses and dynamical masses estimated above for all three clouds are very much consistent with those quantities for the faintest Im galaxies in Virgo for which we have $\mathrm{H}$ I detections (Hoffman et al. 1987).

\subsection{Interaction Scenarios and Group Mass Estimates}

At a cursory glance, neither the $\mathrm{H}$ I distribution of NGC 4532, taken in isolation, nor that of DDO 137 (both shown in Fig. 4) alerts the viewer to the complex interactions within the system. Only upon much closer inspection does one note the asymmetry of the $\mathrm{H}$ I around NGC 4532, with a more rapid decrease in column density toward the north than to the south; the flare to the east does not reveal itself until one inspects the individual channel maps (Fig. 1) or the velocity field (Fig. 5a). Another possible explanation for the asymmetrical $\mathrm{H}$ I distribution around NGC $4532 \mathrm{might}$ be ram pressure by the Virgo Cluster intracluster medium (Phookun \& Mundy 1995). However, the galaxy is not close to any X-ray-emitting portion of the cluster complex or to any particularly dense subcluster. Furthermore, the absorption seen against a continuum source toward the north end of the optical image is a clue that some unusual starburst is going on (LHS98). It seems more likely that the disturbance is caused somehow by interactions within the NGC 4532/ DDO 137 system. Ram pressure against diffuse $\mathrm{H}$ I within the system is a possibility, but the well-separated gas concentrations and strange kinematics of the diffuse gas lead us to the more likely possibility that a clumpy dark matter halo enveloping the entire system is driving the motions and concentration of the gas. Neither the column density map (Fig. 4b) nor the velocity field (Fig. 5b) of DDO 137 is unusual for an irregular galaxy; it is only in the larger context of a map showing DDO 137 and cloud B along with the plume curving to the southwest that one realizes that the slight linear extension toward the northwest of DDO 137 may in fact be a stream. There is, however, no hint of a stellar tidal stream emanating from either galaxy in the $B$ or $R$-band CCD images from LHS98, shown in Figure $4 b$. What might be taken for a tidal tail stretching southeast from NGC 4532 in the DSS image of the POSS-I plate is revealed to be only a string of individual foreground stars on the POSS-II film and in our CCD frames.

Could another galaxy be involved? There is no other cataloged galaxy with known redshift less than $3000 \mathrm{~km} \mathrm{~s}^{-1}$ within $34^{\prime}$. The nearest large galaxy is NGC 4570, an $\mathrm{S0}_{1}(7) / \mathrm{E} 7, B_{T}=11.84$, galaxy at $62^{\prime}$ (more than 5 times the separation of DDO 137 from NGC 4532) and $V_{\odot}=1730$ $\mathrm{km} \mathrm{s}^{-1}$ (RC3). On the Digitized Sky Survey it appears to be a classic edge-on $\mathrm{S} 0$ with no hint of tidal interaction.

Could some portion of the extended gas distribution be produced by a blowout from the intense starburst in NGC 4532 (e.g., Meurer, Staveley-Smith, \& Killeen 1998)? A blowout toward us along the line of sight would account for the blueshifted velocities of clouds A and C. However, gas is not expected to achieve escape velocity from a galaxy as massive as this (Mac Low \& Ferrara 1998), and the blowout would more likely occur perpendicular to the disk of the galaxy, which we see nearly edge-on. The gas would also have to have time to collect and condense into the observed clouds, which is unlikely to happen while the starburst is still underway. In any case, we would be left with the question of what provided the trigger for the starburst.

We see no hint of starlight at our $R$ sensitivity limit of 26 mag $\operatorname{arcsec}^{-2}$ at the positions of the clouds in any of the other CCD images (LHS98); the $B$-band images are shown in Figure 11a, 11b, and 11c. (We take the off-center smudge in cloud A to be a background galaxy; it is indistinguishable from the many other background galaxy "smudges" throughout the frame.) Are these gas concentrations nascent dwarf galaxies, eventually to form stars as in the tidal

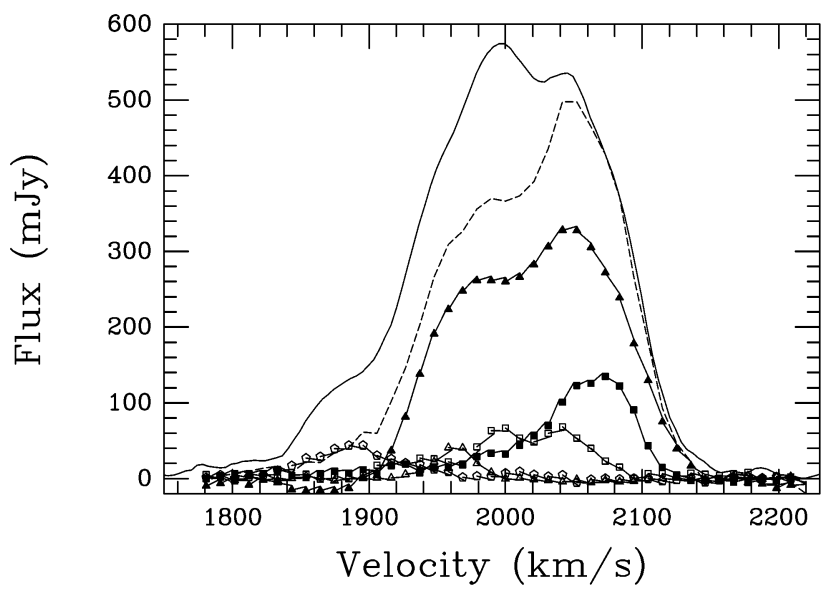

FIG. 14.-Global spectrum of the NGC 4532/DDO 137 system integrated over the Arecibo map (solid curve) with VLA contributions from each of the discrete components indicated: NGC 4532 (filled circles), DDO 137 (filled squares), and clouds A, B, and C (open triangles, open squares, and pentagons, respectively). The dashed curve is the sum of the VLA spectra for the five discrete components. 
streams studied by Mirabel et al. (1992), Elmegreen et al. (1993), Duc \& Mirabel (1994), Hibbard et al. (1994), Hunsberger et al. (1996), and Malphrus et al. (1997)? The H I masses reported in the preceding section are appropriate to observed dwarf star-forming galaxies (Salpeter \& Hoffman 1996; Roberts \& Haynes 1994), but the H I mass alone does not appear to be sufficient to gravitationally bind any of the three clouds if they are currently in virial equilibrium. Dwarf galaxies formed from tidal debris are not expected to have significant dark matter halos (Barnes \& Hernquist 1992b; Elmegreen et al. 1993). The central column densities are (5-6) $\times 10^{20}$ atoms cm $\mathrm{cm}^{-2}$ for each, close to the critical column density for star formation (Kennicutt 1989), but our surface brightness limits are close to the central surface brightnesses of the most extreme LSB galaxies known (O'Neil, Bothun, \& Cornell 1997), and more than $2 \mathrm{mag}$ $\operatorname{arcsec}^{-2}$ fainter than most LSB galaxies.

We can estimate the mass of the group of five objects (two galaxies and three clouds) on the assumption that the system is in virial equilibrium either by taking each of the five as a point object tracer of the gravitational potential of the group, using a standard virial estimator (e.g., Binney \& Tremaine 1987) or by measuring the velocity width of the global $\mathrm{H}$ I profile. Both approaches give consistent results: a group mass in the range (3-6) $\times 10^{11} M_{\odot}$ (see Table 5). This is approximately an order of magnitude larger than the sum of the dynamical masses of all the components, estimated from their rotation curves or $\mathrm{H}$ I profile widths. Table 5 also reports the total stellar mass of each luminous component, calculated from the $R$ magnitude with RC3 corrections for internal and Galactic extinction, using the disk $M / L_{R}$ from our rotation curve fitting. The gas mass of each distinct component is also tabulated; it is calculated from the $\mathrm{H}$ I mass (VLA measurement for each galaxy or cloud, Arecibo measurement for the system as a whole) by multiplying by a factor of $4 / 3$ to account for primordial Helium gas. We have ignored any contribution from molecular gas. Unless the virial estimates of the group mass are deceiving us by more than a factor of a few, the dynamics of the group are controlled by dark matter, which lies mostly outside the objects we can see.

In a similar vein, the total $\mathrm{H}$ I mass of the group as measured at Arecibo is larger by $60 \%$ than the sum of the $\mathrm{H}$ I masses of the two galaxies as measured by the VLA-in other words, the extragalactic gas amounts to $75 \%$ of the gas remaining in NGC 4532, or more than 3 times the gas remaining in DDO 137 , and $28 \%$ of the total $\mathrm{H}$ I lies outside all of the galaxies and discrete gas concentrations. Furthermore, as we noted in HLS + 93, the bulk of the extragalactic gas - clouds A and C along with the southwestern part of the plume stretching out from DDO 137 and the diffuse material extending both east and west from NGC 4532, visible in the Arecibo map but only hinted at in the VLA maps presented here-falls at lower velocity than either of the two galaxies. Only cloud B lies at the velocity of NGC 4532, below that of DDO 137 to which it is apparently connected. Yet these features are spread out in all directions around the two galaxies. Any one of these features appearing at lower velocity than its associated galaxy would not be surprising; a tidal stream that achieved escape velocity mainly along the line of sight would appear blueshifted. But it would be highly improbable for the aggregate - one plume, two discrete clouds and a large quantity of diffuse gas - all to be blueshifted if they were produced by tides acting between the two galaxies. Nor is it likely that any tidal scenario can account for such a large fraction of the gas having been removed from the galaxies.

Sancisi (1998) has suggested that when a disk galaxy shows signs of recent interaction but has no companion galaxy of sufficient size visible today a companion may have been present before the interaction but has now dispersed (or has been incorporated into the larger disk). Hunter et al. (1998) make a similar suggestion for NGC 4449. Because of the large quantity of very extended gas present in our system, we are instead led to speculate that the supposed tidal features are the result of the interaction between one large Sm galaxy (NGC 4532) and a large H I cloud resembling H I 1225 + 01 (Giovanelli et al. 1991), each with a large complement of dark matter (or perhaps in a common halo of dark matter). The dark matter haloes are now overlapping, with the more diffuse $\mathrm{H}$ I cloud having been disrupted in the process; star formation has proceeded only in the most dense clump (DDO 137) of the fragmented cloud. A star formation history analysis of CCD imaging in $U B V R I$ and $\mathrm{H} \alpha$ is underway (LHS98). Clouds A, B and C may either be manifestations of the tidal interaction between the original $\mathrm{H}$ I cloud and the clumpy dark matter potential, or the result of gas falling into dense clumps of dark matter within the common halo. High-resolution simulations show that such clumps can survive for significant times within group scale halos if their internal densities are sufficiently high (Klypin et al. 1997; Tormen, Bouchet, \& White 1997a; Tormen, Diaferio, \& Syer 1997b; Ghigna et al. 1998).

\section{SUMMARY}

Neutral hydrogen synthesis array mapping of the extended gas cloud around the widely separated galaxy pair NGC 453 /DDO 137 reveals three $\mathrm{H}$ I concentrations well separated from the two main galaxies. The $\mathrm{H}$ I masses and indicative gravitational masses are consistent with those of faint Im galaxies in the Virgo Cluster (Hoffman et al. 1987). No stars are visible within these clouds on the CCD images of $\mathrm{Lu}$ et al. (1998). The inferred dynamical mass of each cloud, assuming virial equilibrium, exceeds the $\mathrm{H}$ I mass by factors of 4-20. The central column density of each cloud is close to the critical column density for star formation (Kennicutt 1989), but our upper limits on surface brightness are comparable to the central surface brightnesses of the most extreme LSB galaxies known (O'Neil et al. 1997).

The $\mathrm{H}$ I distribution and rotation curve of NGC 4532 display some peculiarities consistent with tidal interaction; those of DDO 137 are more nearly in accord with expectations for a low-mass irregular galaxy. A maximum-disk hypothesis applied to fitting exponential star + gas disks to the observed rotation curves would require very different mass-to-light ratios for the stars in NGC 4532 and those in DDO 137, perhaps consistent with their very different apparent star formation activities ( $\mathrm{Lu}$ et al. 1998). The shapes of the rotation curves of DDO 137 and of the approaching side of NGC 4532 are consistent with a constant ratio of dark matter to star + gas density at all observed radii in each; however, if the receding side of NGC 4532 is the one less severely afflicted with noncircular velocities (as suggested by all other indications of tidal interaction in the galaxy), then the usual dark matter halo is required.

All available estimators of the total mass of the group (consisting of two galaxies plus three extragalactic clouds) 
give a total mass that is larger by an order of magnitude than the sum of the masses of the individual objects out to the largest radii measured in the VLA maps, as estimated from their rotation curves or virial analyses. The $\mathrm{H}$ i fluxes reported in Hoffman et al. (1993) for NGC 4532 and DDO 137 were not properly corrected for beam overlaps; we give the corrected fluxes in Table 4. (The error affected only NGC 4532/DDO 137, not the other galaxies discussed in that earlier paper.) After correction, the total flux at Arecibo still exceeds the sum of the $\mathrm{H}$ I masses of the two galaxies as measured in the VLA mapping by $37 \%$, and it exceeds the sum of $\mathrm{H}$ I masses of all discrete components (including clouds A, B, and C) by $28 \%$. These facts, along with the kinematic peculiarities of the system, lead us to advance the hypothesis that the system progenitor was a bound pair consisting of the large Sm galaxy NGC 4532 and a large star-poor gas cloud similar to H I $1225+01$, each domi- nated by dark matter. The dark matter halos have now merged, and gas is being accreted into the densest clumps within the common dark matter halo. Star formation has proceeded only in the two densest clumps-NGC 4532, which probably had stars prior to the merger, and DDO 137, which perhaps had no prior stars. Detailed star formation history analysis to follow (Lu et al. 1998) should help us to constrain this scenario further.

We thank J. H. Loh and A. Rabbani for assistance with some preliminary data reductions and the staff of the Very Large Array for their assistance in acquiring the $\mathrm{H}$ I synthesis data. The anonymous referee's voluminous comments led to substantial improvements in our analysis and presentation. This work was supported in part by National Science Foundation grants AST 90-15181 and AST 9316213 at Lafayette College
Bahcall, J. N., et al. 1996, ApJ, 457, 19

Barnes, J. E., \& Hernquist, L. 1992a, AR\&A, 30, 705 $1992 \mathrm{~b}$, Nature, 360, 715 1996, ApJ, 471, 115

Binggeli, B., Sandage, A., \& Tammann, G. A. 1985, AJ, 90, 1681

Binney, J., \& Tremaine, S. 1987, in Galactic Dynamics, ed. J. Binney \& S. Tremaine (Princeton: Princeton Univ. Press), 610

Bottema, R. 1997, A\&A, 328, 517

Bowen, D. V., Blades, J. C., \& Pettini, M. 1996, ApJ, 464, 141

Bruzual A, G., \& Charlot, S. 1993, ApJ, 405, 538

Chengalur, J. N., Salpeter, E. E., \& Terzian, Y. 1994, AJ, 107, 1984 1995, AJ, 110, 167

Connell, B. 1998, BS thesis, Lafayette College

de Vaucouleurs, G., de Vaucouleurs, A., Corwin, H. G., Jr., Buta, R. J., Paturel, G., \& Fouqué, P. 1991, Third Reference Catalog of Bright Galaxies (New York: Springer) (RC3)

Dubinski, J., Mihos, J. C., \& Hernquist, L. 1996, ApJ, 462, 576

Duc, P.-A., \& Mirabel, I. F. 1994, A\&A, 289, 83

Elmegreen, D. M., Kaufman, M., Brinks, E., Elmegreen, B. G., \& Sundin, M. 1995a, ApJ, 453, 100

Elmegreen, B. G., Kaufman, M., \& Thomasson, M. 1993, ApJ, 412, 90

Elmegreen, B. G., Sundin, M., Kaufman, M., Brinks, E., \& Elmegreen, D. M. 1995b, ApJ, 453, 139

Ghigna, S., Moore, B., Governato, F., Lake, G., Quinn, T., \& Stadel, J. 1998, MNRAS, 300, 146

Giovanelli, R., Williams, J. P., \& Haynes, M. P. 1991, AJ, 101, 1242

Haynes, M. P. 1981, AJ, 86, 1126

Haynes, M. P., Giovanelli, R., \& Roberts, M. S. 1979, ApJ, 229, 83

Helou, G., Hoffman, G. L., \& Salpeter, E. E. 1984, ApJS, 55, 433

Hibbard, J. E., Guhathakurta, P., van Gorkom, J. H., \& Schweizer, F. 1994, AJ, 107, 67

Hibbard, J. E., \& van Gorkom, J. H. 1996, AJ, 111, 655

Hoffman, G. L., Helou, G., Salpeter, E. E., Glosson, J., \& Sandage, A. 1987, ApJS, 63, 247

Hoffman, G. L., Lu, N. Y., Salpeter, E. E., Connell, B. M., \& FromholdTreu, R. 1998, ApJ, 500, 789

Hoffman, G. L., Lu, N. Y., Salpeter, E. E., Farhat, B., Lamphier, C., \& Roos, T. 1993, AJ, 106, 39 (HLS + 93)

Hoffman, G. L., Salpeter, E. E., Lamphier, C., \& Roos, T. 1992, ApJ, 388, L5

Howard, S., Keel, W. C., Byrd, G., \& Burkey, J. 1993, ApJ, 417, 502

Hunsberger, S. D., Charlton, J. C., \& Zaritsky, D. 1996, ApJ, 462, 50

Hunter, D. A., Wilcots, E. M., van Woerden, H., Gallagher, J. S., \& Kohle, S. 1998, ApJ, 495, L47

Kaufman, M., Brinks, E., Elmegreen, D. M., Thomasson, M., Elmegreen, B. G., Struck, C., \& Klarić, M. 1997, AJ, 114, 2323

\section{EFERENCES}

Kennicutt, R. C., Jr. 1989, ApJ, 344, 685

Klypin, A., Gottlöber, S., Kravtsov, A. V., \& Khokhlov, A. M. 1997, ApJ, submitted (astro-ph/9708191)

Le Brun, V., Bergeron, J., \& Boissé, P. 1996, A\&A, 306, 691

Li, J. G., \& Seaquist, E. R. 1994, AJ, 107, 1953

Lu, N. Y., Hoffman, G. L., Groff, T., Roos, T., \& Lamphier, C. 1993, ApJS, 88,383

Lu, N. Y., Hoffman, G. L., \& Salpeter, E. E. 1998, in preparation (LHS98)

Mac Low, M.-M., \& Ferrara, A. 1998, ApJ, submitted (astro-ph/9801237)

Malphrus, B. K., Simpson, C. E., Gottesman, S. T., \& Hawarden, T. G. 1997, AJ, 114, 1427

Meurer, G. R., Staveley-Smith, L., \& Killeen, N. E. B. 1998, MNRAS, 300, 705

Mihos, J. C., Dubinski, J., \& Hernquist, L. 1998, ApJ, 494, 183

Mihos, J. C., \& Hernquist, L. 1996, ApJ, 464, 641

Mirabel, I. F., Dottori, H., \& Lutz, D. 1992, A\&A, 256, L19

Mo, H. J., \& Morris, S. L. 1994, MNRAS, 269, 52

Morris, S. L., \& van den Bergh, S. 1994, ApJ, 427, 696

Morris, S. L., Weymann, R. J., Dressler, A., McCarthy, P. J., Smith, B. A., Terrile, R. J., Giovanelli, R., \& Irwin, M. 1993, ApJ, 419, 524

Nordgren, T. E., Chengalur, J. N., Salpeter, E. E., \& Terzian, Y. 1997a, AJ, 114,77

. 1997b, AJ, 114, 913

O’Neil, K., Bothun, G. D., \& Cornell, M. E. 1997, AJ, 113, 1212

Phookun, B., \& Mundy, L. G. 1995, ApJ, 453, 154

Phookun, B., Mundy, L. G., Teuben, P. J., \& Wainscoat, R. J. 1992, ApJ, 400, 516

Roberts, M. S., \& Haynes, M. P. 1994, AR\&A, 32, 115

Salpeter, E. E., \& Hoffman, G. L. 1996, ApJ, 465, 595

Sancisi, R. 1998, in IAU Symp. 186, Galaxy Interactions at Low and High Redshifts, ed. D. Sanders \& J. Barnes (Dordrecht: Kluwer), in press

Schneider, S. E. 1989, ApJ, 343, 94

Smith, B. J., Struck, C., \& Pogge, R. W. 1997, ApJ, 483, 754

Sofue, Y. 1994, ApJ, 423, 207

Stengler-Larrea, E. A., et al. 1995, ApJ, 444, 64

Storrie-Lombardi, L. J., McMahon, R. G., Irwin, M. J., \& Hazard, C. 1994, ApJ, 427, L13

Toomre, A., \& Toomre, J. 1972, ApJ, 178, 623

Tormen, G., Bouchet, F. R., \& White, S. D. M. 1997a, MNRAS, 286, 865

Tormen, G., Diaferio, A., \& Syer, D. 1997b, MNRAS, 299, 728

van Driel, et al. 1992, A\&A, 259, 71

van Gorkom, J. 1993, in The Environment and Evolution of Galaxies, ed.

J. M. Shull \& H. A. Thronson (Dordrecht: Kluwer), 345

van Moorsel, G. A. 1988, A\&A, 202, 59

Yun, M. S., Ho, P. T. P., \& Lo, K. Y. 1994, Nature, 372, 530 